\title{
Variational Problems in the Theory of Elliptic Partial Differential Equations*
}

\author{
P. R. GARABEDIAN \\ Stanford University, Stanford, California \\ $\&$ \\ M. SCHIFFER \\ Stanford University, Stanford, California
}

Communicated by S. BerganaN

1. Introduction. In various problems of mathematical physics one is led to study functionals of closed curves or surfaces, such as, for example, the electrostatic capacity, the virtual mass, the eigenvalues of certain boundary value problems, etc. In order to obtain estimates for these functionals in terms of simpler geometric quantities or of other functionals, we study extremum problems for these expressions. It is now a remarkable fact that in almost all cases where an exact solution of an extremum problem can be given, the solution curve or surface has analytic character. In this paper we wish to develop through a few examples of general interest some mathematical tools by means of which the existence of analytic extremal curves for certain of these minimum problems involving linear elliptic partial differential equations with analytic coefficients and in two independent variables can be verified. Our main interest in the approach to such problems which we present here arises from its wide applicability to a varied field of classical questions in elliptic partial differential equations. We treat in detail only a few special variational problems for the eigenvalues of the vibrating membrane, but it is our hope that the reader will find in our concrete discussion of such examples the outline of the general principles which are the main center of attraction.

There are a few basic ideas involved in our variational and analytical investiga-

* Research under the sponsorship of the Office of Naval Research. 
tions of which a survey at this point might be of some service to the reader. For the existence of extremal configurations, we rely on the standard notions of equicontinuity and of convergence of domains which are by this time familiar in the theory of conformal mapping. We obtain in such a manner quite general extremal curves whose preliminary differentiability properties are studied by means of certain level curve variations based on Schwarz's inequality, by means of the concept of symmetrization as it has been developed by Pólya \& Szegö [17], and by means of the monotonicity of the domain functionals of which we treat. Techniques of interior variation which have been investigated extensively in conformal mapping $[\mathbf{5}, \mathbf{7}, \mathbf{1 9}, \mathbf{2 0}]$ are brought into play in such a way that they can be applied to the extremal functions so obtained, and we are thus able to present a variational theory adequate for the rigorous study of elliptic partial differential equations.

The final step in the treatment, after suitable variational formulas and identities have been set up, involves a proof of the analyticity of the extremal curves. This step is carried through here by means of a set of non-linear integral equations whose derivation is based on the theory of the Riemann function and the problem of Cauchy for linear partial differential equations in the complex domain. We solve the integral equations by successive approximations to determine certain analytic functions whose existence guarantees analyticity of the extremal boundary. This procedure has already proved to be quite useful in hydrodynamical problems [6, 14].

A theory in the calculus of variations of the type which we develop in this report might prove to be quite useful for the systematic treatment of a large number of problems in applied mathematics. Our specific results lead in every case to the final statement that there exists an analytic arc, satisfying a variational condition, which bounds an extremal domain associated with minimizing some combination of principal frequency, area, or length, etc. A short survey of further problems in hydrodynamics, vibration theory, and elasticity for which our theory is relevant should indicate the scope of the paper.

2. Theory of the membrane equation. In a plane, simply-connected region $D$ with boundary curve $C$, we consider the partial differential equation

$$
\Delta u+\lambda u=0
$$

of the eigenfunctions of the vibrating membrane. There exists a sequence of real eigenvalues $\lambda_{n}, 0<\lambda_{1} \leqq \lambda_{2} \leqq \lambda_{3} \leqq \cdots \leqq \lambda_{n} \leqq \cdots$, with corresponding eigenfunctions $u_{1}, u_{2}, \cdots u_{n}, \cdots$ which are solutions of (2.1) in $D$ subject to the boundary condition $u=0$ on $C$, and which satisfy the orthonormality conditions

$$
\iint_{D} u_{i} u_{j} d x d y=\delta_{i j}=\left\{\begin{array}{ll}
0, & i \neq j \\
1, & i=j
\end{array} .\right.
$$


The equation (2.1) has a fundamental solution of the form

$$
S(x, y ; \xi, \eta)=J_{0}\left(\lambda^{\frac{1}{2}} r\right) \log \frac{1}{r}+\text { entire function of } r^{2},
$$

where $r^{2}=(x-\xi)^{2}+(y-\eta)^{2}$, and where

$$
J_{0}(z)=\sum_{n=0}^{\infty} \frac{(-1)^{n} z^{2 n}}{2^{2 n}(n !)^{2}}
$$

is the Bessel function of order zero. We shall make use in the following of the relations $J_{0}(0)=1, J_{0}^{\prime}(0)=0$, in particular. If $\Gamma$ is any smooth closed curve in $D$, then inside $\Gamma$ we can express any solution $u$ of $(2.1)$ in the form

$$
u(\xi, \eta)=\frac{1}{2 \pi} \int_{\Gamma}\left\{u \frac{\partial}{\partial \nu} S(x, y ; \xi, \eta)-S(x, y ; \xi, \eta) \frac{\partial u}{\partial \nu}\right\} d s,
$$

where $s$ is arc length and $\nu$ is the inner normal along $\Gamma$. The expression on the right is inside $\Gamma$ a regular function of $\xi$ and $\eta$, and it can be continued analytically into the domain of two complex variables $\xi$ and $\eta$. If we set $\zeta=\xi+i \eta, \zeta^{*}=$ $\xi-i \eta$ and note that

$$
r^{2}=(z-\zeta)\left(\bar{z}-\zeta^{*}\right),
$$

we see that the analytic continuation of $u(\xi, \eta)$ given by the explicit representation (2.4) extends to all values of $\xi$ and $\eta$ for which both $\zeta$ and $\bar{\zeta}^{*}$ lie in $D$. In particular, the eigenfunctions $u_{n}$ can be continued analytically into the complex domain in this manner.

Considered as a function of the two independent complex variables $\zeta$ and $\zeta^{*}$, $u$ satisfies because of (2.1) the second order differential equation

$$
\frac{\partial^{2} u}{\partial \zeta \partial \zeta^{*}}=-\frac{\lambda}{4} u \text {. }
$$

The differentiation with respect to the complex variables $\zeta, \zeta^{*}$ can be expressed in terms of the differential operators in $\xi$ and $\eta$, if we put

$$
\frac{\partial}{\partial \zeta}=\frac{1}{2}\left(\frac{\partial}{\partial \xi}-i \frac{\partial}{\partial \eta}\right), \quad \frac{\partial}{\partial \zeta^{*}}=\frac{1}{2}\left(\frac{\partial}{\partial \xi}+i \frac{\partial}{\partial \eta}\right) .
$$

In order to obtain a more useful formula for the analytic continuation of $u$ into the domain of the two independent complex variables $\zeta$ and $\zeta^{*}$ which depends only on the values of $u$ and its derivatives on an arc between $\zeta$ and $\bar{\zeta}^{*}$, 
we note that by Stokes' theorem and $\left(2.1^{\prime}\right)$

$$
\begin{gathered}
\int\left\{\frac{\partial}{\partial z^{*}} J_{0}\left(\lambda^{\frac{3}{3}}[z-\zeta]^{\frac{1}{3}}\left[z^{*}-\zeta^{*}\right]^{\frac{3}{3}}\right) u d z^{*}+J_{0}\left(\lambda^{\frac{3}{3}}[z-\zeta]^{\frac{1}{3}}\left[z^{*}-\zeta^{*}\right]^{\frac{1}{3}}\right) \frac{\partial u}{\partial z} d z\right\} \\
=\iint\left\{u \frac{\partial^{2} J_{0}}{\partial z \partial z^{*}}+\frac{\partial J_{0}}{\partial z^{*}} \frac{\partial u}{\partial z}-\frac{\partial J_{0}}{\partial z^{*}} \frac{\partial u}{\partial z}-J_{0} \frac{\partial^{2} u}{\partial z \partial z^{*}}\right\} d z d z^{*} \\
=\left(-\frac{\lambda}{4}\right) \iint\left\{u J_{0}-J_{0} u\right\} d z d z^{*}=0 .
\end{gathered}
$$

Hence the line integral on the left is independent of path in the 4-dimensional space of two independent complex variables $z=x+i y, z^{*}=x-i y$. The integral vanishes if it is evaluated over a closed path consisting of a rectilinear segment from $\bar{\zeta}^{*}, \zeta^{*}$ to $\zeta, \zeta^{*}$, a rectilinear segment from $\zeta, \zeta^{*}$ to $\zeta, \bar{\zeta}$, and a path in the real domain $D$ of $x$ and $y$ from $\zeta, \bar{\zeta}$ to $\bar{\zeta}^{*}, \zeta^{*}$. The integral over the segment from $\zeta, \zeta^{*}$ to $\zeta, \bar{\zeta}$ vanishes, since $z \equiv \zeta$ on this path, the integral over the segment from $\bar{\zeta}^{*}, \zeta^{*}$ to $\zeta, \zeta^{*}$ yields because $z^{*}=\zeta^{*}$ there and $J_{0}(0)=1$ the value $u\left(\zeta, \zeta^{*}\right)-$ $u\left(\bar{\zeta}^{*}, \zeta^{*}\right)$, and thus we have altogether

$$
\begin{aligned}
& u\left(\zeta, \zeta^{*}\right)=u\left(\bar{\zeta}^{*}, \zeta^{*}\right)+\int_{\bar{\zeta}^{*}, \zeta^{*}}^{\zeta \cdot \bar{\zeta}}\left\{\frac{\partial}{\partial \bar{z}} J_{0}\left(\lambda^{\frac{1}{3}}[z-\zeta]^{\frac{1}{3}}\left[\bar{z}-\zeta^{*}\right]^{\frac{3}{3}}\right) u d \bar{z}\right. \\
& \left.+J_{0}\left(\lambda^{\frac{1}{3}}[z-\zeta]^{\frac{1}{1}}\left[\bar{z}-\zeta^{*}\right]^{\frac{3}{3}}\right) \frac{\partial u}{\partial z} d z\right\}
\end{aligned}
$$

where

$$
\frac{\partial}{\partial z}=\frac{1}{2}\left(\frac{\partial}{\partial x}-i \frac{\partial}{\partial y}\right), \quad \frac{\partial}{\partial \bar{z}}=\frac{1}{2}\left(\frac{\partial}{\partial x}+i \frac{\partial}{\partial y}\right) .
$$

This is the desired formula for $u$ at the point $\xi, \eta$ (which we have also characterized by the coordinates $\left.\zeta, \zeta^{*}\right)$ in terms of the values of $u$ and its first derivatives in the real domain $D$. The function $J_{0}\left(\lambda^{\frac{1}{3}}[z-\zeta]^{\frac{3}{3}}\left[z^{*}-\zeta^{*}\right]^{\frac{3}{3}}\right)$ is the well-known Riemann function [3] for the equation $\left(2.1^{\prime}\right)$ in the complex domain. Equation (2.5) is merely the explicit representation by means of the Riemann function of the solution $u$ of the Cauchy problem for (2.1) with initial data given along a curve from $\zeta$ to $\bar{\zeta}^{*}$ in the real domain.

Each eigenfunction $u_{n}$ for (2.1) satisfies a homogeneous integral equation

$$
u_{n}(t, \bar{t})=\frac{\lambda_{n}}{2 \pi} \iint_{D} u_{n}(z, \bar{z}) G(z, \bar{z} ; t, \bar{t}) d x d y,
$$

where

$$
G(z, \bar{z} ; t, \bar{t})=\log \frac{1}{|z-t|}+\cdots
$$


is the Green's function of Laplace's equation in $D$. For a convex region $D$, we can use (2.6) to show that the gradient of $u_{n}$ is bounded in $D$. Let us suppose at first that $C$ is a smooth convex curve. Then the normal derivative of $u_{n}$ on $C$ can be written

$$
\frac{\partial u_{n}}{\partial \nu}=\frac{\lambda_{n}}{2 \pi} \iint_{D} u_{n} \frac{\partial G}{\partial \nu} d x d y
$$

at any point $t$ on $C$. We denote by $L_{t}$ the tangent to $C$ at $t$ and we let $g_{t}$ be the Green's function for Laplace's equation in the half-plane containing $D$ bounded by $L_{t}$. Then by the maximum principle $g_{t} \geqq G$ in $D$, and hence

$$
\frac{\partial G}{\partial \nu} \leqq \frac{\partial g_{t}}{\partial \nu}
$$

at $t$. By Schwarz's inequality and the normalization of $u$,

$$
\left|u_{n}\right| \leqq \frac{\lambda_{n}}{2 \pi}\left[\iint_{D} G^{2} d x d y\right]^{1} \leqq \frac{\lambda_{n}}{2 \pi}\left[\iint_{D} g_{t}^{2} d x d y\right]^{\frac{1}{2}}
$$

is bounded, and hence

$$
\left|\frac{\partial u_{n}}{\partial \nu}\right| \leqq\left(\frac{\lambda_{n}}{2 \pi}\right)^{2}\left[\max \iint_{D} g_{t}{ }^{2} d x d y\right]^{\frac{1}{2}} \iint_{D} \frac{\partial g_{t}}{\partial \nu} d x d y .
$$

Thus the normal derivative $\partial u_{n} / \partial \nu$ is bounded on $C$ by a number which can be estimated in terms of $\lambda_{n}$ alone.

In order to estimate $\partial u_{n} / \partial z$ in $D$, we substitute $u_{n}$ into (2.4) with $\Gamma$ replaced by $C$ and differentiate. This gives, since $u_{n}=0$ on $C$,

$$
\frac{\partial u_{n}}{\partial z}=\frac{1}{2 \pi} \int_{C}\left\{\frac{\partial}{\partial t} S(\xi, \eta ; \sigma, \tau)-\frac{\partial}{\partial z} S(\xi, \eta ; x, y)\right\} \frac{\partial u_{n}}{\partial \nu} d s
$$

where $t=\sigma+i \tau$ is any point outside $C$. It is easily seen from (2.8) that there is a constant $M_{n}$ such that

$$
\left|\frac{\partial u_{n}}{\partial z}\right| \leqq M_{n} \int_{c} \frac{|z-t|}{|z-\zeta||t-\zeta|} d s .
$$

We choose $t$ to lie as close as possible to $z$, with the restriction that the line bisecting the segment from $z$ to $t$ does not intersect $D$. With this choice of $t$, the integral on the right in (2.9) can be estimated independently of $C$ and $z$, due to the convexity of the path of integration. Hence we obtain a bound for $\partial u_{n} / \partial z$ in terms of $\lambda_{n}$ alone.

The case of a convex region with a boundary for which the above integrations of normal derivatives are not justified can be treated by approximation with 
smoothly bounded convex domains, since the estimates obtained for the smooth domains depend only on $\lambda_{n}$ and not directly on the geometry of $C$. Thus we verify that $\partial u_{n} / \partial z$ is bounded in any convex region.

Let $u$ be an arbitrary solution of $(2.1)$ and let $\omega(x, y)$ be a fixed non-vanishing solution of the same differential equation in some domain $\Omega$. It can then easily be verified that the expression

$$
v(x, y)=\frac{u(x, y)}{\omega(x, y)}
$$

satisfies in $\Omega$ the partial differential equation

$$
\frac{\partial}{\partial x}\left(\omega^{2} v_{x}\right)+\frac{\partial}{\partial y}\left(\omega^{2} v_{y}\right)=0
$$

Thus, the second order equation (2.1) can always be reduced to a system of first order equations

$$
\omega^{2} v_{x}=w_{y}, \quad \omega^{2} v_{y}=-w_{x}
$$

in a sufficiently small domain $\Omega$.

We may use, for example, the particular function

$$
\omega=\cos \left[\lambda^{\frac{3}{2}}\left(x-x_{0}\right)\right]
$$

in the vicinity of any given point $t_{0} \equiv\left(x_{0}, y_{0}\right)$ and we are led to

$$
v=\frac{u}{\cos \left[\lambda^{\frac{3}{2}}\left(x-x_{0}\right)\right]},
$$

which satisfies together with its conjugate function $w$ the system

$$
v_{x} \cos ^{2}\left[\lambda^{\frac{1}{2}}\left(x-x_{0}\right)\right]=w_{y}, \quad v_{y} \cos ^{2}\left[\lambda^{\frac{1}{2}}\left(x-x_{0}\right)\right]=-w_{x} .
$$

Let $u(x, y)$ be a solution of (2.1) which vanishes on $C$. If $t_{0} \equiv\left(x_{0}, y_{0}\right)$ is an arbitrary boundary point of $D$, we may construct a function $v(x, y)$ which will satisfy (2.10) in the intersection of $D$ with a domain $\left|z-t_{0}\right|<c$ for $c$ small enough. We have $v=0$ on the arc of $C$ in $\Omega$ and, if we assume

$$
\iint_{D}\left(u_{x}^{2}+u_{y}^{2}\right) d x d y<\infty
$$

we can also assert that

$$
\iint_{\Omega}\left(w_{x}^{2}+w_{y}{ }^{2}\right) d x d y=\iint_{\Omega}\left(w_{r}{ }^{2}+\frac{1}{r^{2}} w_{\phi}^{2}\right) r d r d \phi<\infty,
$$

where $r, \phi$ are polar coordinates about a boundary point $t_{1}$ of $C$ in $\Omega$. 
Let us suppose now that the complex-valued function $v+i w$ is univalent in $\Omega$; we shall then prove that $w$ is continuous at the boundary point $t_{1}$ of $C$ in $\Omega$.

If $w$ were not continuous at $t_{1}$, we could find a curve $\Gamma$ in $D$ ending at $t_{1}$ along which $v+i w$ does not approach a limit. Clearly $v \rightarrow 0$ as $z \rightarrow t_{1}$ along $\Gamma$, and thus $w$ must oscillate between its lower and upper limits, $w_{1}$ and $w_{2}$. Let $\Gamma_{1}$ and $\Gamma_{2}$ be the inverse images in the $z$-plane of the lines $w=w_{1}+\eta, w=w_{2}-\eta$, for $0<\eta<\frac{1}{2}\left(w_{2}-w_{1}\right)$. Then $\Gamma_{1}$ and $\Gamma_{2}$ intersect $\Gamma$ infinitely often near $t_{1}$.

Now let $\gamma_{r}$ be the intersection of $\Omega$ with the circle $\left|z-t_{1}\right|=r$, and notice that

$$
V_{r}^{2}=\left(\int_{\gamma_{r}}\left|w_{\phi}\right| d \phi\right)^{2} \leqq 2 \pi \int_{\gamma_{r}} w_{\phi}^{2} d \phi
$$

by Schwarz's inequality. Hence, for $R$ sufficiently small,

$$
\int_{0}^{R} \frac{V_{r}^{2}}{r} d r \leqq 2 \pi \int_{0}^{R} \int_{\gamma_{r}} \frac{1}{r^{2}} w_{\phi}^{2} r d \phi d r \leqq 2 \pi \iint_{\Omega}\left(w_{r}{ }^{2}+\frac{1}{r^{2}} w_{\phi}^{2}\right) r d r d \phi<\infty,
$$

and it follows that the variation $V_{r}$ of $w$ on $\gamma_{r}$ approaches zero as $r \rightarrow 0$ through a suitable sequence of values. But this contradicts the statement that $\Gamma_{1}$ and $\Gamma_{2}$ intersect $\Gamma$ near $t_{1}$ infinitely often, since in this case $\Gamma_{1}$ and $\Gamma_{2}$ would have also to intersect all the $\operatorname{arcs} \gamma_{r}$. Hence, $w$ must, indeed, be continuous.

3. Variational formulas. We should like to show that the eigenvalues $\lambda_{n}$ depend continuously on the domain $D$ and that the corresponding eigenfunctions $u_{n}$ have the same property in some generalized sense. In order to do this, we notice that by virtue of the general theory of the integral equation (2.6),

$$
\frac{2 \pi}{\lambda_{n}}=\max \iiint \int G(z, \bar{z} ; t, \bar{t}) U(z, \bar{z}) U(t, \bar{t}) d x d y d \sigma d \tau
$$

for $U$ in the class of functions satisfying the normalization conditions

$$
\iint U^{2} d x d y=1, \quad \iint U u_{k} d x d y=0, \quad k=1,2,3, \cdots, n-1 .
$$

Here we can take as domain of integration the entire plane, provided that we define the Green's function $G$ and the eigenfunctions $u_{k}$ to be zero outside $D$. The eigenfunction $u_{n}$ is the extremal function $U$ for (3.1).

Let $D^{*}$ be a region converging towards $D$ in the Fréchet sense and let $G^{*}$, $u_{n}{ }^{*}$, and $\lambda_{n}{ }^{*}$ be the corresponding Green's function, eigenfunctions and eigenvalues. Clearly

$$
\left|\iiint \int\left(G-G^{*}\right) U U d x d y d \sigma d \tau\right|^{2} \leqq \iiint \int\left(G-G^{*}\right)^{2} d x d y d \sigma d \tau=I
$$


by Schwarz's inequality and (3.2), and hence

$$
\left|2 \pi / \lambda_{1}-2 \pi / \lambda_{1}^{*}\right| \leqq I^{\frac{1}{2}}
$$

Also, the integral $I \rightarrow 0$, and hence $\lambda_{1}{ }^{*} \rightarrow \lambda_{1}$. Furthermore, the eigenfunctions $u_{1}^{*}$ of the domains $D^{*}$ are uniformly bounded by an estimate of the form (2.7), and therefore, according to the normalization (2.2), $u_{1}{ }^{*}$ does not approach zero in $D$. Hence $\lambda_{1}{ }^{*}$ tends to the lowest principal frequency $\lambda_{1}$ of $D$, and $u_{1}{ }^{*}$ approaches the eigenfunction $u_{1}$ uniformly in each of its closed subregions. Indeed, it follows from the integral equation (2.6) that the functions $u_{1}^{*}$ form an equicontinuous family of functions in such closed subregions, and they converge to a normalized, non-negative solution of (2.1) as a result. But in addition, the Green's functions $G^{*}$ converge uniformly to $G$ in $D$, by the maximum principle. Hence the integral equation (2.6) is satisfied by the limit of $u_{1}{ }^{*}$, which is therefore identical with $u_{1}$.

The higher eigenvalues $\lambda_{n}{ }^{*}$ and eigenfunctions $u_{n}{ }^{*}$ of $D^{*}$ are likewise seen to converge to eigenvalues $\lambda_{m}$ and eigenfunctions $u_{m}$ of $D$.

Let us consider next the first order change in the eigenvalues $\lambda_{n}$ with a variation of the domain $D$ caused by making an infinitesimal shift $\delta \nu$ of the curve $C$, assumed now to be analytic, along its inner normal $\nu$ with respect to $D$. We denote the varied domain, curve, eigenvalues and eigenfunctions by $D^{*}, C^{*}$, $\lambda_{n}{ }^{*}$ and $u_{n}{ }^{*}$, respectively. We assume, at first, that $\lambda_{n}$ is a non-degenerate eigenvalue and that $D^{*} \supset D$; we suppose $D^{*}$ so near to $D$ that $u$ is still analytic in $D^{*}$ and that $\lambda_{n}{ }^{*}$ is still non-degenerate. The corresponding eigenfunctions $u_{n}$ and $u_{n}{ }^{*}$ are, in this case, uniquely determined. By Green's theorem

$$
\begin{aligned}
& \iint_{D} \nabla u_{n} \nabla u_{n}^{*} d x d y+\iint_{D} u_{n} \Delta u_{n}^{*} d x d y+\int_{C} u_{n} \frac{\partial u_{n}^{*}}{\partial \nu} d s=0, \\
& \iint_{D^{*}} \nabla u_{n} \nabla u_{n}^{*} d x d y+\iint_{D^{*}} u_{n}^{*} \Delta u_{n} d x d y+\int_{C^{*}} u_{n}^{*} \frac{\partial u_{n}}{\partial \nu} d s=0,
\end{aligned}
$$

and, since $\Delta u_{n}=-\lambda_{n} u_{n}$ in $D, \Delta u_{n}{ }^{*}=-\lambda_{n}{ }^{*} u_{n}{ }^{*}$ in $D^{*}, u_{n}=0$ on $C, u_{n}^{*}=0$ on $C^{*}$, subtraction of these formulas gives

$$
\begin{array}{rl}
\iint_{D} \nabla u_{n} \nabla u_{n}^{*} d x d y-\iint_{D^{*}} \nabla u_{n} \nabla u_{n} * & d x d y \\
& =\lambda_{n} * \iint_{D} u_{n} u_{n}^{*} d x d y-\lambda_{n} \iint_{D^{*}} u_{n} u_{n}^{*} d x d y
\end{array}
$$

For small shifts $\delta \nu, u_{n}$ and $u_{n}{ }^{*}$ differ very little, and near $C$ and $C^{*}, u_{n}$ and $u_{n}{ }^{*}$ 
differ very little from 0 , respectively. Hence to within accuracy of the first order in $\delta \nu$,

$$
\int_{C}\left(\nabla u_{n}\right)^{2} \delta \nu d s=\left(\lambda_{n}^{*}-\lambda_{n}\right) \iint_{D} u_{n}^{2} d x d y .
$$

Since $u_{n}=0$ on $C$, and by virtue of the normalization (2.2), we obtain the final variational formula $[\mathbf{9}, \mathbf{1 8}]$

$$
\delta \lambda_{n}=\lambda_{n} *-\lambda_{n}=\int_{c}\left(\frac{\partial u_{n}}{\partial \nu}\right)^{2} \delta \nu d s .
$$

We have established (3.4), so far, only in the case that $D^{*} \supset D$, that is, for $\delta \nu<0$. For the general case, we may compare the eigenvalues $\lambda_{n}$ and $\lambda_{n}{ }^{*}$ with the eigenvalues $\lambda_{n}{ }^{+}$of a domain $D^{+}$which is also near to $D$ and contains both $D$ and $D^{*}$. Applying (3.4) once with respect to $D$ and $D^{+}$and then with respect to $D^{*}$ and $D^{+}$, we can easily derive the formula (3.4) for the general case.

The formula (3.4) is no longer valid in the case of a multiple eigenvalue $\lambda_{n}=$ $\lambda_{n+1}=\cdots=\lambda_{m}$, since we cannot conclude that $u_{n}{ }^{*}$ is near $u_{n}$. We deduce here, by a reasoning which will begiven in the sequel for a more useful type of variation, that the variations $\delta \lambda_{n}=\lambda_{n}{ }^{*}-\lambda_{n}, \cdots, \delta \lambda_{m}=\lambda_{m}{ }^{*}-\lambda_{m}$ are, to within accuracy of the first order, the eigenvalues of the matrix of quantities

$$
\int_{C} \frac{\partial u_{k}}{\partial \nu} \frac{\partial u_{l}}{\partial \nu} \delta \nu d s
$$

with $k$ and $l$ running from $n$ to $m$, inclusive. The variations $\delta \lambda_{k}$ are positive for positive shifts $\delta \nu$, since the quadratic form

$$
\sum\left\{\int_{C} \frac{\partial u_{l}}{\partial \nu} \frac{\partial u_{k}}{\partial \nu} \delta \nu d s\right\} \xi_{l} \xi_{k}=\int_{C}\left\{\sum \frac{\partial u_{k}}{\partial \nu} \xi_{k}\right\}^{2} \delta \nu d s
$$

is positive-definite. One can verify from (3.4) and (3.5) that the eigenvalues $\lambda_{n}$ increase steadily as the domain $D$ diminishes, a property which is also apparent from the integral equation (2.5) and the monotonicity of the Green's function in dependence on $D$.

Because of the formal nature of the derivation of (3.4), it is desirable in the applications to have a variational formula for the eigenvalues $\lambda_{n}$ which can be used for more general domains $D$. For this purpose we let $F(z, \bar{z})$ be any complexvalued function with derivatives of all orders, and for small complex values of the parameter $\epsilon$ we consider the transformation

$$
w=z+\epsilon F(z, \bar{z}) \text {. }
$$


This function maps $D$ on a neighboring region $D^{*}$. We suppose that

$$
\lambda=\lambda_{n}=\lambda_{n+1}=\cdots=\lambda_{m}
$$

is an eigenvalue of (2.1) in $D$ of multiplicity $m-n$ and we let $\lambda_{n}{ }^{*}, \cdots, \lambda_{m}{ }^{*}$ be the corresponding eigenvalues of $D^{*}$, with which are associated eigenfunctions $u_{n}{ }^{*}, \cdots, u_{m}{ }^{*}$. Because of the continuous dependence of the eigenfunctions on the domain, we can write

$$
u_{j}^{*}(w, \bar{w})=\sum_{k=n}^{m} \alpha_{j k} u_{k}(z, \bar{z})+o(1)
$$

where the $\alpha_{j k}$ are real and depend on $F$ and $\epsilon$. Treating $u_{j}^{*}$ as a function of $w, \bar{w}$ (and thus by (3.6) as a function of $z, \bar{z}$ ) and $u_{k}$ as a function of $z, \bar{z}$, we find

$$
\begin{aligned}
0 & =\int_{C}\left\{u_{k} \frac{\partial u_{j}^{*}}{\partial \nu}-u_{j}^{*} \frac{\partial u_{k}}{\partial \nu}\right\} d s \\
& =\iint_{D}\left\{u_{j}^{*} \Delta u_{k}-u_{k} \Delta u_{j}^{*}\right\} d x d y
\end{aligned}
$$

with

$$
\Delta=4 \frac{\partial^{2}}{\partial z \partial \bar{z}}
$$

Since

$$
\begin{aligned}
\Delta u_{k} & =-\lambda u_{k}, \\
\Delta u_{j}^{*} & =-\lambda_{j}^{*} u_{j}^{*}-2 \operatorname{Re}\left\{\epsilon \frac{\partial F}{\partial z} u_{j}^{*} \lambda_{j}^{*}-\epsilon \Delta F \frac{\partial u_{j}^{*}}{\partial w}-4 \epsilon \frac{\partial F}{\partial \bar{z}} \frac{\partial^{2} u_{j}^{*}}{\partial w^{2}}\right\}+O\left(\epsilon^{2}\right),
\end{aligned}
$$

this yields

$$
\begin{aligned}
& \left(\lambda-\lambda_{j}^{*}\right) \iint_{D} u_{k} u_{j}^{*} d x d y \\
& \quad=2 \operatorname{Re}\left\{\iint_{D} u_{k}\left[\epsilon \frac{\partial F}{\partial z} u_{j}^{*} \lambda_{j}^{*}-\epsilon \Delta F \frac{\partial u_{j}^{*}}{\partial w}-4 \epsilon \frac{\partial F}{\partial \bar{z}} \frac{\partial^{2} u_{j}^{*}}{\partial w^{2}}\right] d x d y\right\}+O\left(\epsilon^{2}\right) .
\end{aligned}
$$

On the other hand,

$$
\iint u_{k} u_{j}^{*} d x d y=\alpha_{j k}+o(1)
$$


and therefore

$$
\begin{aligned}
\alpha_{j k}\left(\lambda-\lambda_{j}^{*}\right)=2 \operatorname{Re}\left\{\epsilon \sum _ { l = n } ^ { m } \alpha _ { j l } \int \int _ { D } u _ { k } \left[\lambda u_{l} \frac{\partial F}{\partial z}-\frac{\partial u_{l}}{\partial z} \Delta F\right.\right. & \\
& \left.\left.-4 \frac{\partial^{2} u_{l}}{\partial z^{2}} \frac{\partial F}{\partial \bar{z}}\right] d x d y\right\}+o(\epsilon) .
\end{aligned}
$$

By an easy integration by parts, we then obtain

$$
\begin{aligned}
\sum_{l=n}^{m} \alpha_{j l} \operatorname{Re}\left\{\epsilon \iint_{D}\left[4 \frac{\partial u_{k}}{\partial z} \frac{\partial u_{l}}{\partial z} \frac{\partial F}{\partial \bar{z}}+\lambda u_{k} u_{l} \frac{\partial F}{\partial z}\right] d x d y\right\}+o(\epsilon) & \\
& =\frac{1}{2} \alpha_{j k}\left(\lambda-\lambda_{j}^{*}\right), \quad k=n, \cdots, m .
\end{aligned}
$$

Since not all the $\alpha_{j l}$ vanish, the variations $\delta \lambda_{j}=\lambda_{j}{ }^{*}-\lambda_{j}$ are, to within accuracy of the first order in $\epsilon$, the eigenvalues of the matrix of elements

$$
-2 \operatorname{Re}\left\{\epsilon \iint_{D}\left[4 \frac{\partial u_{k}}{\partial z} \frac{\partial u_{l}}{\partial z} \frac{\partial F}{\partial \bar{z}}+\lambda u_{k} u_{l} \frac{\partial F}{\partial z}\right] d x d y\right\}
$$

with $k$ and $l$ running from $n$ to $m$, inclusive. In particular, for a simple eigenvalue $\lambda_{n}$, we obtain the elegant variational formula

$$
\delta \lambda_{n}=-2 \operatorname{Re}\left\{\epsilon \iint_{D}\left[4\left(\frac{\partial u_{n}}{\partial z}\right)^{2} \frac{\partial F}{\partial \bar{z}}+\lambda_{n} u_{n}{ }^{2} \frac{\partial F}{\partial z}\right] d x d y\right\}+o(\epsilon)
$$

Let us observe that we can derive from (3.8) a new proof for the formula (3.4) which has been established previously in a rather heuristic way. In fact, let us assume that $C$ is a smooth curve; then we have by integration by parts

$$
\begin{aligned}
\iint_{D}\left[4\left(\frac{\partial u}{\partial z}\right)^{2} \frac{\partial F}{\partial \bar{z}}+\lambda u^{2} \frac{\partial F}{\partial z}\right] d x d y=\frac{1}{2 i} \int_{C} F & \left\{4\left(\frac{\partial u}{\partial z}\right)^{2} d z-\lambda u^{2} d \bar{z}\right\} \\
& -\iint_{D} F\left[4 \frac{\partial}{\partial \bar{z}}\left(\frac{\partial u}{\partial z}\right)^{2}+\lambda \frac{\partial}{\partial z} u^{2}\right] d x d y .
\end{aligned}
$$

Because of the differential equation $\left(2.1^{\prime}\right)$ the last integrand vanishes identically. On the other hand, we have on $C$

$$
u=0, \quad \frac{\partial u}{\partial z} \frac{d z}{d s}=\frac{i}{2} \frac{\partial u}{\partial \nu}
$$


Hence, (3.8) may be transformed into

$$
\delta \lambda_{n}=\int_{c}\left(\frac{\partial u}{\partial \nu}\right)^{2} \operatorname{Re}\left\{\frac{\epsilon F}{i \frac{d z}{d s}}\right\} d s+o(\epsilon) .
$$

It is easily verified that

$$
\delta \nu=\operatorname{Re}\left\{\frac{\epsilon F}{i \frac{d z}{d s}}\right\}
$$

represents the normal shift of the boundary points under (3.6). Thus (3.4) has been proved again for this wide class of variations.

Special cases of $F$ in (3.8) prove to be quite useful. For example, let $\omega$ be a function which is 1 in an arbitrary fixed region $\Omega$, which vanishes outside a neighborhood of $\Omega$ and which has derivatives of all orders. For $t$ outside $D$, set

$$
F(z, \bar{z})=\frac{\omega}{z-t}
$$

For $t$ inside $D$ we have to change the definition of $F(z, \bar{z})$ near $t$ in order to preserve the regular character of $F$. Let $\rho$ be so small that the circle $|z-t| \leqq$ $\rho$ lies inside $D$ and set

$$
\begin{array}{ll}
F(z, \bar{z})=\frac{\omega}{z-t}, & |z-t| \geqq \rho, \\
F(z, \bar{z})=\frac{\omega}{z-t}\left[1-\exp \frac{(z-t)(\bar{z}-\bar{t})}{(z-t)(\bar{z}-\bar{t})-\rho^{2}}\right], & |z-t|<\rho .
\end{array}
$$

It is obvious that $F$ has continuous derivatives of all orders in $D$. We denote by $\delta(t)$ the function which is 1 in $D$ and 0 outside $D$, and we obtain from (3.8) after easy calculations by letting $\rho \rightarrow 0$

$$
\begin{aligned}
\delta \lambda_{n}=-8 \pi \operatorname{Re}\left\{\epsilon\left(\frac{\partial u_{n}}{\partial t}\right)^{2} \delta(t)\right\}+2 \operatorname{Re}\left\{\epsilon \lambda_{n} \iint_{\Omega \cdot D} \frac{u_{n}^{2} d x d y}{(z-t)^{2}}\right\} & +\operatorname{Re}\{\epsilon Q(t)\}+o(\epsilon),
\end{aligned}
$$

where $Q(t)$ is an analytic function of $t$ in $\Omega$. Formula (3.9) gives the variation of $\lambda_{n}$ for the shift of the boundary $C$ of $D$ by an amount $\frac{\epsilon \omega}{z-t}$. This variational formula depends only upon the values which $F$ assumes on $C$ and is independent of the smoothing of $F$ near the point $t$. We might also have chosen

$$
F(z, \bar{z})=\omega \frac{\bar{z}-\bar{t}}{\rho^{2}} \text { for }|z-t|<\rho
$$


in the above definition. Here $F$ would not have had continuous derivatives of all orders in $D$, but it is easy to verify directly that (3.9) holds in this case, too.

Similarly, formulas for the variation of multiple eigenvalues can be obtained with this special function $F$. In particular, for the multiple eigenvalue $\lambda=\lambda_{n}=$ $\cdots=\lambda_{m}$, we find

$$
\begin{aligned}
\delta\left\{\sum_{j=n}^{m} \lambda_{j}\right\}=-8 \pi & \operatorname{Re}\left\{\sum_{j=n}^{m} \epsilon\left(\frac{\partial u_{j}}{\partial t}\right)^{2} \delta(t)\right\} \\
& +2 \operatorname{Re}\left\{\epsilon \sum_{j=n}^{m} \lambda_{j} \iint_{D} \frac{u_{j}^{2} d x d y}{(z-t)^{2}}\right\}+\operatorname{Re}\{\epsilon Q(t)\}+o(\epsilon)
\end{aligned}
$$

since the trace of the symmetric matrix (3.7) is equal to the sum of its eigenvalues.

4. Length extremal problem for the membrane. Let $L$ denote the length of the boundary $C$ of the membrane $D$. It is known that the dimensionless product $L^{2} \lambda_{1}$ is a minimum when $D$ is a circle [2]. We shall discuss in this section the nature of a membrane $D$ for which the product $L^{2} \lambda_{2}$ is a minimum.

Given a region $D$, let $D^{*}$ be its convex hull. That is, let $D^{*}$ be the smallest convex region containing $D$. It is clear that the length $L^{*}$ of the perimeter of $D^{*}$ is smaller than the length $L$ of $C$ if $D$ is not itself convex. Also, by the monotonicity of $\lambda_{2}$ in dependence on $D$, derived from (3.4) and (3.5), it is seen that the second frequency $\lambda_{2}{ }^{*}$ of $D^{*}$ is smaller than $\lambda_{2}$, since $D^{*}$ includes $D$. Thus $L^{2} \lambda_{2}>$ $L^{*^{2}} \lambda_{2}{ }^{*}$, and we conclude that there is no loss of generality if we consider only convex membranes $D$ in competition for the extremal problem

$$
L^{2} \lambda_{2}=\text { minimum. }
$$

For (4.1), we normalize $D$ so that it is contained within the unit circle, but not within any smaller circle. This is possible, since $L^{2} \lambda_{2}$ is unchanged by translation, rotation or magnification of $D$. The convex curves $C$ bounding such membranes $D$ can be expressed in parametric form so as to yield an equicontinuous family of functions. Thus, by the continuity of the eigenfunctions and eigenvalues in dependence on the domain, established in Section 3, we see that an extremal domain $D$ for (4.1) exists, unless $D$ degenerates to a segment in the minimal case.

That this latter possibility is excluded can again be seen from the monotonic dependence of $\lambda_{2}$ on $D$. For a convex curve $C$ which approximates a linear slit can be included in a long narrow rectangle $R$ whose second frequency is of the order of magnitude of the inverse square of its width. The rectangle $R$ has a smaller second frequency than $D$, and hence for long, narrow regions $D$ of the type described, the product $L^{2} \lambda_{2}$ is arbitrarily large. 
Thus a non-degenerate extremal domain $D$ for (4.1) can be found. We apply to it a variation of the type (3.6) with $F$ of the special form

$$
F(z, \bar{z})=\frac{1}{z-t}
$$

if $t$ lies in $D$ we have to smooth $F(z, \bar{z})$ near $t$ in an analogous way as in $\left(3.6^{\prime}\right)$. This yields for $L$ the variation

$$
\delta L=-\operatorname{Re}\left\{\epsilon \int_{c} \frac{d s}{(z-t)^{2}}\right\}+o(\epsilon) .
$$

Let us suppose, at first, that the second eigenvalue of the extremum domain $D$ is non-degenerate and that, therefore, formula (3.9) is applicable. Because of the minimum property (4.1) of $D$ we have

$$
2 L \lambda_{2} \delta L+L^{2} \delta \lambda_{2} \geqq 0,
$$

and from the arbitrariness of $\epsilon$ in (3.9) and (4.2) this yields

$$
\lambda_{2} \int_{C} \frac{d s}{(z-t)^{2}}+4 \pi L \delta(t)\left(\frac{\partial u_{2}}{\partial t}\right)^{2}-L \lambda_{2} \iint_{D} \frac{u_{2}^{2} d x d y}{(z-t)^{2}}=0 .
$$

In (4.4) we take the region $\Omega$ of (3.9) to be $D$, and it can easily be seen that in this case $Q(t) \equiv 0$ in (3.9).

For the area integral on the right in (4.4) we have by Green's theorem

$$
\iint_{D} \frac{u_{2}^{2} d x d y}{(z-t)^{2}}=2 \iint_{D} \frac{\partial u_{2}}{\partial z} \frac{u_{2} d x d y}{(z-t)}
$$

Since $D$ is convex, $\partial u_{2} / \partial z$ is bounded, and the integral on the right is a continuous function of $t$ across $C$. Hence the boundary values of $\left(\partial u_{2} / \partial t\right)^{2}$ on $C$ can be found from (4.4) in terms of the jump of $\int_{C}(z-t)^{-2} d s$ across $C$.

We set $\dot{z}=\partial z / \partial s=e^{i \theta}$ along $C$, and we note that

$$
\int_{C} \frac{d s}{(z-t)^{2}}=\int_{C} \frac{\overline{\dot{z}} d z}{(z-t)^{2}}=\int_{C} \frac{d \overline{\dot{z}}}{z-t} .
$$

Let $t_{1}$ and $t_{2}$ be points near $C$ inside $D$ and let $w_{1}$ and $w_{2}$ be points outside $C$, near $t_{1}$ and $t_{2}$, respectively. From (4.4) we see that as $t_{1}$ and $w_{1}$ approach $z_{1}$ on $C$ and as $t_{2}$ and $w_{2}$ approach $z_{2}$ on $C$,

$$
\frac{4 \pi L}{\lambda_{2}} \int_{t_{1}}^{t_{2}}\left(\frac{\partial u_{2}}{\partial t}\right)^{2} d t+\int_{c} \log \left[\frac{\left(z-t_{1}\right)\left(z-w_{2}\right)}{\left(z-t_{2}\right)\left(z-w_{1}\right)}\right] d \overline{\dot{z}}
$$

approaches zero. 
Passing to the limit, we find

$$
\left|\int_{z_{1}}^{z_{2}} d \overline{\dot{z}}\right|=\left|\int_{z_{1}}^{z_{2}} d e^{-i \theta}\right| \leqq M \int_{z_{1}}^{z_{2}} d s
$$

where $M$ is an upper bound for $\left(2 L / \lambda_{2}\right)\left(\partial u_{2} / \partial t\right)^{2}$. Hence $\theta=\theta(s)$ satisfies on $L$ a Lipschitz condition $\left|\theta\left(s_{2}\right)-\theta\left(s_{1}\right)\right| \leqq M_{0}\left|s_{2}-s_{1}\right|$.

We see that the curvature $\kappa=\dot{\theta}(s)=\partial \theta / \partial s$ must exist almost everywhere along $C$ and must be bounded there. Let $z_{1}$ be a point on $C$ where $\kappa$ exists, let $t$ and $w$ be points inside and outside $D$, respectively, on the normal to $C$ at $z_{1}$, and suppose $\left|t-z_{1}\right|=\left|w-z_{1}\right|=\rho$. By (4.4) we must have

$$
\lim _{t \rightarrow z_{1}} \frac{2 L}{\lambda_{2}}\left(\frac{\partial u_{2}}{\partial t}\right)^{2}=\lim _{\rho \rightarrow 0} \frac{-1}{\pi} \int_{c} \frac{\overline{\dot{z}}-\overline{\dot{z}}_{1}}{z-z_{1}} \frac{(t-w)\left(z-z_{1}\right)^{2}}{(z-t)^{2}(z-w)^{2}} d z
$$

where $\dot{z}_{1}=e^{i \theta_{1}}$ is the unit tangent at $z_{1}$. We use here the fact that

$$
\frac{1}{2 \pi i} \int_{c} \frac{z-z_{1}}{(z-t)^{2}(z-w)^{2}} d z=\frac{2 z_{1}-(t+w)}{(t-w)^{3}}=0
$$

in view of the relation between $z_{1}, t$ and $w$. On the other hand, obviously

$$
\lim _{\rho \rightarrow 0} \frac{-1}{\pi} \int_{c} \frac{\overline{\dot{z}}_{1}\left(-i_{\kappa_{1}}\right)}{\dot{z}_{1}} \frac{(t-w)\left(z-z_{1}\right)^{2}}{(z-t)^{2}(z-w)^{2}} d z=-\dot{\vec{z}}_{1}^{2} \kappa_{1},
$$

where $\kappa_{1}$ is the curvature $\kappa=\dot{\theta}$ at $z_{1}$. But, if we measure arc length $s$ from $z_{1}$,

$$
\frac{\overline{\dot{z}}-\overline{\dot{z}}_{1}}{z-z_{1}}+i_{\kappa_{1}} \overline{\dot{z}}_{1}^{2}=o(1)
$$

as $s \rightarrow 0$. Hence

$$
\left|\int_{C}\left[\frac{\overline{\dot{z}}-\overline{\dot{z}}_{1}}{z-z_{1}}+i_{\kappa_{1}}{\overline{\dot{z}}_{1}^{2}}^{2}\right] \frac{(t-w)\left(z-z_{1}\right)^{2}}{(z-t)^{2}(z-w)^{2}} d z\right|
$$

$$
\leqq M_{1} \int_{0}^{\rho} \frac{o(1) s^{2} d s}{\rho^{3}}+M_{1} \int_{\rho}^{\sqrt{\rho}} \frac{\rho o(1) d s}{s^{2}}+M_{1} \int_{\sqrt[V]{ } \bar{\rho}}^{\infty} \frac{\rho d s}{s^{2}},
$$

for some large value of $M_{1}$. The integrals on the right in (4.6) tend to zero as $\rho \rightarrow 0$, and hence by (4.5), for $z$ on $C$,

$$
\lim _{t \rightarrow z}\left(\frac{\partial u_{2}}{\partial t}\right)^{2}=-\frac{\lambda_{2} \kappa \bar{z}^{2}}{2 L}
$$

almost everywhere. 
From (4.7) it follows easily that

$$
\lim _{t \rightarrow z} \frac{\partial u_{2}}{\partial t}= \pm i \sqrt{\frac{\lambda_{2}}{2 L}} \kappa^{\frac{1}{2} \bar{z}}
$$

exists also almost everywhere on $C$. We want to show next that $C$ can be decomposed into at most two arcs on each of which one definite sign holds. We consider the subdomains $D_{+}$and $D_{-}$of $D$ in which $u_{2}$ is positive or negative, respectively; there are exactly two such domains since $u_{2}$ is the second eigenfunction of $D$. Let $C_{+}$and $C_{-}$be the $\operatorname{arcs}$ of $C$ which belong to the boundary of $D_{+}$and $D_{-}$. Since $u_{2}=0$ on $C_{1}$ we have

$$
\lim _{b \rightarrow z} \int_{a}^{b} d u_{2}=-u_{2}(a)>0
$$

if $z$ lies in $C_{-}$and $a$ in $D_{-}$. On the other hand,

$$
\int_{a}^{b} d u_{2}=2 \operatorname{Re}\left\{\int_{a}^{b} \frac{\partial u_{2}}{\partial t} d t\right\}
$$

This shows, clearly, that we have to choose on $C_{-}$the upper sign in $\left(4.7^{\prime}\right)$ and on $C_{+}$the lower sign.

We shall utilize $\left(4.7^{\prime}\right)$ in order to express the analytic function $\partial u_{2}(z, \zeta) / \partial z$ in terms of $\kappa$ by means of formula (2.5). We substitute $u_{2}$ for $u$ in (2.5), differentiate with respect to $\zeta$, and replace $\zeta$ by $t$ to obtain

$$
\frac{\partial}{\partial t} u_{2}\left(t, \zeta^{*}\right)=\frac{\partial}{\partial t} u_{2}(t, \bar{t})+\int_{\bar{\zeta}^{*}, \zeta^{*}}^{t, \bar{t}}\left\{\frac{\partial^{2}}{\partial \bar{z} \partial t} J_{0} u_{2} d \bar{z}+\frac{\partial}{\partial t} J_{0} \frac{\partial u_{2}}{\partial z} d z\right\} .
$$

Letting $t$ and $\bar{\zeta}^{*}$ approach positions on $C_{+}$, for example, and letting the path of integration approach $C_{+}$, we obtain from the boundary conditions $u_{2}=0$ and $\left(4.7^{\prime}\right)$, by Lebesgue's convergence theorem,

$$
\left(\frac{2 L}{\lambda_{2}}\right)^{\frac{3}{2}} \frac{\partial}{\partial t} u_{2}\left(t, \zeta^{*}\right)=-i \kappa^{\frac{1}{2}} \bar{t}-i \int_{\bar{\zeta}^{*}, \zeta^{*}}^{t, \bar{t}} \frac{\partial}{\partial t} J_{0}\left(\lambda_{2}^{\frac{1}{2}}[z-t]^{\frac{1}{3}}\left[\bar{z}-\zeta^{*}\right]^{\frac{1}{3}}\right) \kappa^{\frac{1}{2}} d s .
$$

Thus the function

$$
F(t)=\left(\frac{2 L}{\lambda_{2}}\right)^{\frac{1}{2}} \frac{\partial}{\partial t} u_{2}\left(t, \zeta^{*}\right)
$$

is analytic and bounded for $t$ in $D$ and has on $C_{+}$the boundary values (4.8) almost everywhere, for each fixed $\bar{\zeta}^{*}$ on $\mathrm{C}_{+}$. Similarly, $F(t)$ will have almost every- 
where boundary values on $C_{-}$given by an equation analogous to (4.8), if $\xi^{*}$ lies on $C_{-}$also.

Motivated by (4.8), we introduce the system of integral equations

$$
\begin{aligned}
& h(t)=i F(t)-\int_{\bar{\zeta}^{*}, \zeta^{*}}^{t, \bar{i}} \frac{\partial}{\partial t} J_{0}\left(\lambda_{2}^{\frac{1}{2}}[z-t]^{\frac{1}{2}}\left[g(z)-\zeta^{*}\right]^{\frac{1}{2}}\right) h(z) d z, \\
& f(t)=\dot{\zeta}^{*}-i \int_{\bar{\zeta}^{*}, \zeta^{*}}^{t, \bar{t}} h(z)^{2} d z, \\
& g(t)=\zeta^{*}+\int_{\zeta^{*}, \zeta^{*}}^{t, i} f(z)^{2} d z
\end{aligned}
$$

for the determination of the three analytic functions $h(t), f(t)$ and $g(t)$ in $D$. In (4.10), $\dot{\zeta}^{*}$ represents the conjugate of the unit tangent $\dot{z}$ at $\bar{\zeta}^{*}$. Formula (4.8) shows that on $C$,

$$
h=\kappa^{3} \bar{t}, \quad f=\bar{t}, \quad g=\bar{t}
$$

is a bounded solution of the system (4.9), (4.10), (4.11) near $\bar{\zeta}^{*}$.

The Picard method of successive approximations can be applied without difficulty to show that there exists a unique bounded solution $h, f, g$ of (4.9), (4.10), and (4.11) in the neighborhood of $\bar{\zeta}^{*}$ in $D+C$. The successive approximations are chosen to be analytic in $D$ and are independent of the choice of a path of integration from $\bar{\zeta}^{*}$ to $t$, by Cauchy's theorem. They converge uniformly to the solution $h, f, g$, which is therefore analytic in a vicinity of $\bar{\zeta}^{*}$ in $D$ by the Weierstrass convergence theorem $[6,14]$. We shall deal in Section 6 with an analogous system of non-linear integral equations and carry out there the necessary steps in greater detail. We omit the analogous reasoning at this stage in order to avoid repetition and to develop the method in the more complicated form used later on.

The analytic function $g(t)$ is continuous in $D+C$ near $\bar{\zeta}^{*}$, since it is the integral of a bounded function. By the uniqueness of the solution $h, f, g$ of (4.9), (4.10), and (4.11), $g(t)$ must be consistent on $C$ with the known solution (4.12) there. Hence $g(t)$ has the boundary values $g(t)=\bar{t}$ on $C$. We define

$$
\begin{aligned}
& \Phi(t)=t+g(t), \\
& \Psi(t)=t-g(t),
\end{aligned}
$$

and we note that $\Phi$ is real and $\Psi$ is pure imaginary on $C$ near $\bar{\zeta}^{*}$.

We let $t(w)$ be a conformal mapping of the upper half of the $w$-plane onto $D$. On a segment of the real axis in the $w$-plane, $\Phi$ is real and $\Psi$ is imaginary, and 
hence these functions can be continued analytically into the lower half-plane by Schwarz's principle of reflection. In particular,

$$
t=\frac{1}{2}(\Phi+\Psi)
$$

is analytic on the segment of the real axis, and hence the corresponding arc of $C$ is analytic.

Since this reasoning is valid for each interior point $\bar{\zeta}^{*}$ of the arcs $C_{+}$and $C_{-}$, we can conclude that each one of these subarcs is analytic. Let now $\bar{\xi}_{0}^{*}$ be a point of $C$ at which $C_{+}$and $C_{-}$meet. We may set up again the set of non-linear integral equations (4.9), (4.10), and (4.11) and prove the existence of analytic solutions $h, f$ and $g$ near $\bar{\zeta}_{0}^{*}$. We will now find

$$
h= \pm \kappa^{\frac{1}{2}} \bar{t}, \quad f=\bar{t}, \quad g=\bar{t}
$$

where the upper sign holds on $C_{+}$and the lower sign on $C_{-}$. We can show as before that $C$ is analytic near $\bar{\zeta}_{0}^{*}$ and, consequently, that $C$ is a closed analytic curve. The values $\pm \kappa^{\frac{1}{2}} t, \bar{t}, \bar{t}$ appear as the boundary values on $C$ of functions $h$, $f$ and $g$ which are analytic in a neighborhood of the boundary curve $C$.

If we let $t=G(\zeta)$ be the inverse of $\zeta=g(t)$ near $C$, we find from (2.5), (4.7'), and (4.12)

$$
u_{2}\left(t, \zeta^{*}\right)=\left(\frac{\lambda_{2}}{2 L}\right)^{\frac{1}{2}} i \int_{t}^{G\left(\zeta^{*}\right)} J_{0}\left(\lambda_{2}^{\frac{1}{2}}[z-t]^{\frac{1}{2}}\left[g(z)-\zeta^{*}\right]^{\frac{1}{2}}\right) h(z) d z
$$

for $t$ and $\bar{\zeta}^{*}$ on $C$. But by analytic continuation, this formula is seen to be valid for $t$ and $\bar{\zeta}^{*}$ in a complete neighborhood of $C$. Thus

$$
u_{2}(t, \bar{t})=\left(\frac{\lambda_{2}}{2 L}\right)^{\frac{1}{2}} i \int_{t}^{\sigma(\bar{t})} J_{0}\left(\lambda_{2}^{\frac{1}{2}}[z-t]^{\frac{1}{2}}[g(z)-\bar{t}]^{\frac{1}{2}}\right) h(z) d z
$$

is analytic near $C$, and can be continued across $C$ explicitly.

If we set up equation (4.17) formally with arbitrary analytic functions $G(\bar{t})$ and $h(z)$, we obtain a function $u_{2}(t, \bar{t})$ which is a solution of (2.1) and vanishes for $t=G(\bar{t})$, that is, for $\bar{t}=g(t)$. Moreover, one finds easily

$$
\frac{\partial u_{2}(t, \bar{t})}{\partial t}=-i\left(\frac{\lambda_{2}}{2 L}\right)^{\frac{1}{2}} h(t), \quad \text { for } \quad \bar{t}=g(t) .
$$

Thus, if an analytic arc in the complex $t$-plane is given in the form $\bar{t}=g(t)$, one can construct by (4.17) a solution of (2.1) which vanishes along this arc and satisfies there the variational condition (4.7), provided one chooses $h(t)=\kappa^{\frac{3}{3}} t$. The boundary $C$ of the extremum domain $D$ in our problem is distinguished by the global condition that the function $u_{2}(t, \bar{t})$, given by (4.17), be regular inside $C$ and represent the second eigenfunction of the domain considered. 
We proved that the extremal problem (4.1) has a solution $D$ bounded by a simple closed analytic curve $C$. The eigenfunction $u_{2}$ is analytic on $C$, and it satisfies there the variational condition

$$
\left(\frac{\partial u_{2}}{\partial n}\right)^{2}=2 \lambda_{2} \kappa / L
$$

which can be obtained either directly from (4.7) or from (3.4) and the analogous formula

$$
\delta L=-\int_{C} \kappa \delta \nu d s,
$$

valid for the analytic curve $C$.

We have to deal, finally, with another possibility in making these conclusions, namely, that the second eigenvalue might have multiplicity $m-2>0, \lambda_{2}=$ $\lambda_{3}=\cdots=\lambda_{m}$. We shall prove in closing that this eventuality does not occur.

Indeed, for a multiple eigenvalue $\lambda_{2}$, we can replace (4.3) by

$$
2 L \lambda_{k} \delta L+L^{2} \delta \lambda_{k} \geqq 0, \quad k=2, \cdots, m .
$$

Since the numbers $\delta \lambda_{k}$ are the eigenvalues of the matrix (3.7) and since $\epsilon$ has an arbitrary sign in (3.7), we check that for each $k$ between 2 and $m$

$$
\delta \lambda_{k}=-2 \lambda_{k} \delta L / L=-2 \lambda_{2} \delta L / L \text {. }
$$

Thus all the eigenvalues of (3.7) are equal, and this matrix has only zero terms off the main diagonal, while all elements on the main diagonal are equal. Hence (4.4) holds not only for $u_{2}$, but also with $u_{2}$ replaced by any $u_{k}$ with $2 \leqq k \leqq m$. Therefore, finally, (4.18) is valid with $u_{2}$ replaced by each such $u_{k}$, whence, by substituting each $u_{k}$ into (2.4) with $\Gamma$ replaced by $C$, we obtain $u_{2} \equiv u_{3} \equiv \ldots$ $\equiv u_{m}$, a manifest contradiction. Thus, the extremum eigenvalue $\lambda_{2}$ is nondegenerate.

5. Level curve variations. Let us suppose that we are given two simple closed analytic curves $C_{1}$ and $C_{2}$ which can be represented as the graphs of equations $y^{2}=f_{1}(x), y^{2}=f_{2}(x)$, and let us denote by $D_{1}$ and $D_{2}$ the domains bounded by $C_{1}$ and $C_{2}$. Let us suppose further that $D_{2}$ contains the closure of $D_{1}$, and that the principal frequency $\lambda_{1}$ of $D_{1}$ is smaller than the principal frequency $\lambda_{0}$ of the annular region $D_{2}-D_{1}-C_{1}$. We let $C$ be any curve lying between $C_{1}$ and $C_{2}$ and bounding a simply-connected membrane $D$ with area $A$. For the sake of a simpler notation, we shall denote by $\lambda$ and by $u$ the first eigenvalue and first eigenfunction of $D$, without a subscript. For $C_{1}$ and $C_{2}$ fixed, we ask to determine $C$ in such a way that

$$
A \lambda=\text { minimum }
$$


This problem is motivated by the desirability of obtaining an estimate of $\lambda$ in terms of the area $A$ of $D$ when we know that $D$ includes the known domain $D_{1}$ and is included in the known domain $D_{2}$, whose principal frequencies are trivial estimates of $\lambda$. If there is a circle $C$ between $C_{1}$ and $C_{2}$, then this circle solves the extremal problem $(5.1)[4,11,17]$. However, in the general case, $C_{1}$ and $C_{2}$ will not always be separated by a circle, and (5.1) becomes of interest for our variational theory.

A first remark about (5.1) is that symmetrization in the $x$-axis does not alter $D_{1}$ and $D_{2}$, while it can only alter $D$ in such a way as to leave $A$ invariant and to diminish $\lambda$ or leave it unchanged [17]. Thus it is only necessary to allow in the competition for (5.1) curves $C$ bounding domains $D$ which are already symmetrized in the $x$-axis, since any other shapes for $D$ can be replaced by the corresponding symmetrized shapes, which yield smaller values for $A \lambda$.

One can conclude that there exists an extremal domain $D$ for (5.1) as follows. From any sequence of competing domains $D^{(n)}$ with values of $A^{(n)} \lambda^{(n)}$ tending to their greatest lower bound, one can select a subsequence of domains which converges to a kernel domain $D$ containing $D_{1}$. In fact, let $f_{n}(w)$ be the univalent function in $|w|<1$ which maps the unit circle onto the domain $D^{(n)}$ so that $w=0$ corresponds to a fixed point $\alpha$ in $D_{1}$. The functions $f_{n}(w)$ for all competing domains form a normal family and we can select from these functions a subsequence which converges uniformly in $|w| \leqq r<1$. The corresponding domains $D^{(n)}$ will then converge to a kernel domain in the sense of Carathéodory [1]. Furthermore, the eigenfunctions $u^{(n)}$ corresponding to this subsequence of domains form an equicontinuous family of functions in each closed subregion of the limit domain, by virtue of (2.6) and the equicontinuity of the corresponding Green's functions. Hence one can select a further subsequence of the competing domains such that the corresponding eigenfunctions $u^{(n)}$ converge uniformly in each closed subregion of the limit domain to a solution $u$ of (2.1) and of the integral equation

$$
u=\frac{\lambda}{2 \pi} \iint_{D} G u d x d y
$$

Consequently, $\lambda$ will be the first eigenvalue of the limit domain $D$, if we can exclude the possibility that $u \equiv 0$ in the kernel domain $D$.

In order to show that $u \neq 0$ there, we consider a proper subdomain $D_{0}$ of $D_{1}$ with a boundary curve $C_{0}$ which is so close to $C_{1}$ that the ring domain $R=D_{2}-$ $D_{0}-C_{0}$ still has a principal frequency $\lambda^{*}>\lambda_{1}$. This is possible, since we assumed that the principal frequency of $D_{2}-D_{1}-C_{1}$ was larger than $\lambda_{1}$. If $u \equiv 0$, the sequence $u^{(n)}$ of eigenfunctions belonging to the minimal sequence of domains $D^{(n)}$ must converge to zero uniformly in $D_{0}+C_{0}$. We set $u^{(n)}=0$ in $D_{2}-$ $D^{(n)}$, and we denote by $\omega^{(n)}$ a function in the ring $R$ obtained by multiplying 
$u^{(n)}$ with a fixed function which is 1 on $C_{0}$, which vanishes outside a closed curve separating $C_{0}$ and $C_{1}$, and which has bounded derivatives. Clearly

$$
\iint_{R}\left[\nabla \omega^{(n)}\right]^{2} d x d y \rightarrow 0, \quad \omega^{(n)} \rightarrow 0 \text { in } R .
$$

Since $u^{(n)} \rightarrow 0$ uniformly in $D_{0}$, we have also

$$
\iint_{R}\left[u^{(n)}-\omega^{(n)}\right]^{2} d x d y \rightarrow 1, \quad \iint_{R}\left[\nabla\left(u^{(n)}-\omega^{(n)}\right)\right]^{2} d x d y \rightarrow \lambda .
$$

But $u^{(n)}-\omega^{(n)}$ vanishes on the boundary of $R$, and hence

$$
\iint_{R}\left[\nabla\left(u^{(n)}-\omega^{(n)}\right)\right]^{2} d x d y \geqq \lambda^{*} \iint_{R}\left[u^{(n)}-\omega^{(n)}\right]^{2} d x d y
$$

so that $\lambda \geqq \lambda^{*}$. But by assumption, $\lambda^{*}>\lambda_{1}$, and $\lambda_{1} \geqq \lambda$ because $D^{(n)} \supset D_{1}$ and $\lambda_{1} \geqq \lambda^{(n)}$. Thus we arrive at a contradiction, and the sequence $u^{(n)}$ does not converge to zero in $D$. It follows that $\lambda$ is actually the principal frequency of the kernel domain $D$.

It is therefore evident that the limit domain $D$ is extremal for (5.1). Since the competing domains can be assumed to be symmetrized in the $x$-axis without loss of generality, the same follows for $D$.

There is, of course, the possibility that $D$ coincides with the domain $D_{1}$ or with $D_{2}$. In this case, $D$ has an analytic boundary because of our assumptions with respect to $C_{1}$ and $C_{2}$. We want to prove that the boundary $C$ of $D$ is always composed of analytic arcs. The only case of interest occurs when $D$ does not coincide with $D_{1}$ or with $D_{2}$.

We proceed first to show that the boundary $C$ of the extremal domain $D$ is a rectifiable curve. To do this, we let $D_{0}{ }^{*}$ be the region in which $u>\epsilon$, for small $\epsilon>0$ given. We denote by $D^{*}$ the sum $D_{1}+D_{0}^{*}$ and we note that $D^{*}$ is a competing membrane for (5.1). Let $\lambda_{0}{ }^{*}$ and $\lambda^{*}$ be the principal frequencies of $D_{0}{ }^{*}$ and $D^{*}$, respectively, and assume an analogous notation for the other quantities associated with those domains. We find by Green's theorem

$$
\begin{aligned}
\left(\lambda_{0}^{*}-\lambda\right) \iint_{D_{0} *} u u_{0}^{*} d x d y & =\iint_{D_{0}^{*}}\left(u_{0}^{*} \Delta u-u \Delta u_{0}^{*}\right) d x d y \\
& =\iint_{c_{0}^{*}} u \frac{\partial u_{0}^{*}}{\partial \nu} d s \\
& =\epsilon \lambda_{0}^{*} \iint_{D_{0}} u_{0}^{*} d x d y
\end{aligned}
$$


Hence there is a constant $M_{1}$ such that

$$
\delta \lambda=\lambda^{*}-\lambda \leqq \lambda_{0}^{*}-\lambda \leqq M_{1} \epsilon,
$$

since

$$
\iint_{D_{0}^{*}} u u_{0} * d x d y>\frac{1}{2}
$$

for sufficiently small $\epsilon$.

We denote by $C_{\epsilon}$ the arcs of the level curve $u=\epsilon$ which lie in $D_{2}-D_{1}$, and we denote by $A(\epsilon)$ the area of $D^{*}$. We find easily

$$
-\frac{d A}{d \epsilon}=\int_{C_{0}}\left(\frac{\partial u}{\partial \nu}\right)^{-1} d s
$$

whence

$$
-\frac{d A}{d \epsilon} \lambda \iint_{D} u d x d y \geqq \int_{C_{\epsilon}} \frac{\partial u}{\partial \nu} d s \int_{c_{\epsilon}}\left(\frac{\partial u}{\partial \nu}\right)^{-1} d s \geqq\left(\int_{c_{\epsilon}} d s\right)^{2},
$$

by Schwarz's inequality. Hence there is a positive constant $M_{2}$ such that

$$
-\delta A=-\left(A^{*}-A\right)=-\int_{0}^{\epsilon} \frac{d A}{d \epsilon} d \epsilon \geqq M_{2} \int_{0}^{\epsilon}\left(\int_{C_{\epsilon}} d s\right)^{2} d \epsilon .
$$

From the minimum problem (5.1) we have $-\lambda \delta A \leqq A \delta \lambda$, whence by (5.2) and (5.3) we obtain for a suitable large value of $M_{3}$

$$
\int_{0}^{e}\left(\int_{C_{\epsilon}} d s\right)^{2} d \epsilon \leqq M_{3} \epsilon
$$

It follows from (5.4) that we can find a sequence of positive values of $\epsilon$ approaching zero for which the integrals $\int_{c_{\epsilon}} d s$ are bounded. Since $C_{\epsilon}$ approximates the arcs of $C$ in $D_{2}-D_{1}$ for small $\epsilon$ and since length is lower semi-continuous, we conclude that $C$ is rectifiable.

We wish next to apply the variational formula (3.9) to (5.1) with the region $\Omega$ taken to be a small circle in $D_{2}-D_{1}$ about some point of the extremal curve $C$. The corresponding formula for $\delta A$ is

$$
\delta A=\operatorname{Re}\left\{\epsilon i \int_{\gamma} \frac{d_{b} \bar{z}}{z-t}\right\}+Q_{1}(t)+o(\epsilon),
$$


where $Q_{1}(t)$ is analytic in $\Omega$ and where $\gamma$ consists of the arcs of $C$ lying in $\Omega$. Since $\epsilon$ is arbitrary and since by (5.1) $A \delta \lambda+\lambda \delta A=0$, we find

$$
-8 \pi A \delta(t)\left(\frac{\partial u}{\partial t}\right)^{2}+2 A \lambda \iint_{\Omega} \frac{u^{2} d x d y}{(z-t)^{2}}+\lambda i \int_{\gamma} \frac{d \bar{z}}{z-t}+Q_{0}(t)=0,
$$

when $u$ is defined to be zero outside $D$ and where $Q_{0}(t)$ is an analytic function in $\Omega$.

The integral over $\Omega$ in (5.6) can be brought by two applications of Green's theorem into the form

$$
\iint_{\Omega} \frac{u^{2} d x d y}{(z-t)^{2}}=\frac{\lambda}{2} \iint_{\Omega} u^{2} \frac{\bar{z}-\bar{t}}{z-t} d x d y-2 \iint_{\Omega} \frac{\partial u}{\partial z} \frac{\partial u}{\partial \bar{z}} \frac{\bar{z}-\bar{t}}{z-t} d x d y+Q_{2}(t, \bar{t}),
$$

where $Q_{2}(t, \bar{t})$ is an analytic function of $t$ and $\bar{t}$ in $\Omega$. Hence this integral is a continuous function of $t$ throughout $\Omega$. Thus we can show by (5.6) that the limit of $(\partial u / \partial t)^{2}$ as $t$ approaches $\gamma$ is proportional to the jump of the integral $\int_{\gamma} d \bar{z} /(z-t)$ across $\gamma$. This integral can be treated in a manner quite analogous to the analysis leading from (4.4) by means of (4.5) and (4.6) to (4.7). We can show by such an analysis that the limit of $(\partial u / \partial t)^{2}$ exists at all those points of the rectifiable curve $C$ where $\dot{z}=\partial z / \partial s$ exists, and, more precisely, we find that for almost all points $z$ on $C$

$$
\lim _{t \rightarrow z}\left(\frac{\partial u}{\partial t}\right)^{2}=-\frac{\lambda}{4 A} \bar{z}^{2} .
$$

We can also write the extremum condition (5.6) in the form

$$
\frac{\lambda}{4 \pi} \iint_{\Omega} \frac{u^{2}-A^{-1}}{(z-t)^{2}} d x d y=\left(\frac{\partial u}{\partial t}\right)^{2} \delta(t)+Q_{3}(t)
$$

where $Q_{3}$ is a suitable analytic function of $t$ in $\Omega$. We define

$$
\phi(t, \bar{t})=\frac{\lambda}{4 \pi} \iint_{\Omega} \frac{u^{2}-A^{-1}}{z-t} d x d y
$$

and we notice that by the Poisson-Laplace equation

$$
\frac{\partial \phi}{\partial \bar{t}}=-\frac{\lambda}{4}\left(u^{2}-\frac{1}{A}\right) \delta(t)
$$

whereas by (5.8)

$$
\frac{\partial \phi}{\partial t}=\left(\frac{\partial u}{\partial t}\right)^{2} \delta(t)+Q_{3}(t)
$$


For $t$ in $\Omega-D, \phi$ is an analytic function $Q_{4}(t)$, and

$$
\frac{d}{d t} Q_{4}(t)=Q_{3}(t)
$$

On the other hand, in the intersection $\Omega D$ of $\Omega$ and $D$, we can use (5.9) and (5.9') in order to express $\phi$ by the line integral

$$
\phi(t, \bar{t})=\int_{t_{0}, \bar{t}_{0}}^{t_{1} \bar{i}}\left[\left(\frac{\partial u}{\partial z}\right)^{2} d z-\frac{\lambda}{4} u^{2} d \bar{z}\right]+\frac{\lambda}{4 A}\left(\bar{t}-\bar{t}_{0}\right)+Q_{4}(t)
$$

which is independent of path by (2.1).

From its definition, $\phi$ is a continuous function in $\Omega$. Therefore by (5.10) we can write

$$
\int_{t_{0}, \bar{t}_{0}}^{t_{0} i}\left[\left(\frac{\partial u}{\partial z}\right)^{2} d z-\frac{\lambda}{4} u^{2 \cdot} d \bar{z}\right]=-\frac{\lambda}{4 A}\left(\bar{t}-\bar{t}_{0}\right)
$$

for points $t$ and $t_{0}$ on $C$ in $\Omega$, where the integral on the left may also be interpreted to mean the limit of integrals over arcs in $D$ which approximate the arc of $C$ between $t_{0}$ and $t$.

Condition (5.11) is an integrated form of the boundary condition (5.7) which is more useful in the applications because it is identically fulfilled in the sense of continuous limits, whereas (5.7) holds only almost everywhere.

6. Analyticity of the boundary curve. Let us develop for the moment a few heuristic ideas which will help to motivate the material to follow. Let us suppose, to this end, that the arc of the curve $C$ in $\Omega$ is analytic and that we can express $\bar{t}$ on $\gamma$ as an analytic function of $t$, say

$$
\bar{t}=\bar{t}_{0}+g(t), \quad g\left(t_{0}\right)=0 .
$$

From this assumption, we shall be able to derive a functional equation involving $g(t)$, and the analyticity of $\gamma$ will be established conversely by proving that the functional equation possesses an analytic solution $g(t)$ and by identifying, finally, $g(t)$ ou $\gamma$ with $\bar{t}-\bar{t}_{0}$.

It is well known that the function $u(t, \bar{t})$ will be analytic in both arguments if $t, \bar{t}$ lies on the analytic arc $\gamma$. Thus, we have the identity

$$
u\left(t, \bar{t}_{0}+g(t)\right)=0
$$


which holds originally for $t \varepsilon \gamma$, but which must be fulfilled identically for all values $t$. We define further the analytic function

$$
f(t)=u_{t}\left(t, \bar{t}_{0}+g(t)\right) .
$$

We may now express (5.11) in the form

$$
g(t)=-\frac{4 A}{\lambda} \int_{t_{0}}^{t} f(\zeta)^{2} d \zeta, \quad t \varepsilon \gamma, \quad t_{0} \varepsilon \gamma .
$$

A second relation between $g(t)$ and $f(t)$ can be obtained by differentiating (2.5) with respect to $\zeta$ and replacing $\bar{\zeta}^{*}$ by $t_{0}$. This yields, as in Section 4,

$$
\frac{\partial u\left(\zeta, \bar{t}_{0}\right)}{\partial \zeta}=\frac{\partial}{\partial \zeta} u(\zeta, \bar{\zeta})+\int_{t_{0 . t_{0}}}^{\zeta, \bar{\zeta}}\left[\frac{\partial^{2} J_{0}}{\partial \bar{z} \partial \zeta} u d \bar{z}+\frac{\partial J_{0}}{\partial \zeta} \frac{\partial u}{\partial z} d z\right]
$$

This equation holds for all permissible paths of integration between the points $t_{0}, \bar{t}_{0}$ and $\zeta, \bar{\zeta}$. We may impose now the relation $\bar{z}=\bar{t}_{0}+g(z)$ and let $z$ run in $D$ from $t_{0}$ to $\zeta$ along some curve near $C$ where $g(z)$ is analytic. Next, we may apply equations (6.2) and (6.3) and put, therefore, (6.5) into the form

$$
u_{\zeta}\left(\zeta, \bar{t}_{0}\right)=f(\zeta)-\frac{\lambda^{\frac{3}{3}}}{2} \int_{t_{0}}^{\zeta} J_{0}{ }^{\prime}\left\{\lambda^{\frac{1}{2}}[(z-\zeta) g(z)]^{\frac{1}{2}}\right\}\left(\frac{g(z)}{z-\zeta}\right)^{\frac{1}{2}} f(z) d z
$$

Since this identity holds for $\zeta \varepsilon \gamma$, it must hold for all $\zeta$ in a neighborhood of $t_{0}$, and, in particular, in $\Omega \cdot D$. In this domain $u_{\zeta}\left(\zeta, \bar{t}_{0}\right)$ is a known analytic function of $\zeta$ by virtue of the formula (6.5) and we may consider (6.4) and (6.6) as a set of non-linear integral equations for the unknown functions $f(\zeta)$ and $g(\zeta)$.

So far, we were led by heuristic reasoning. Now we set up the non-linear system

$$
\begin{gathered}
u_{\zeta}\left(\zeta, \bar{t}_{0}\right)=f(\zeta)-\int_{0_{0}}^{\zeta} K[\zeta-z, g(z)] f(z) d z \\
g(\zeta)=-\frac{4 A}{\lambda} \int_{t_{0}}^{\zeta} f(z)^{2} d z
\end{gathered}
$$

with

$$
K[\zeta-z, g(z)]=\frac{\lambda^{\frac{3}{3}}}{2} J_{0}^{\prime}\left\{\lambda^{\frac{1}{3}}[(z-\zeta) g(z)]^{\frac{1}{2}}\right\}\left[\frac{g(z)}{z-\zeta}\right]^{\frac{1}{2}}
$$


and we try to solve it directly by successive approximations. We put

$$
\left\{\begin{array}{l}
g_{n}(\zeta)=-\frac{4 A}{\lambda} \int_{t_{0}}^{\zeta} f_{n}(z)^{2} d z \\
f_{n}(\zeta)=u_{\zeta}\left(\zeta, \bar{t}_{0}\right)+\int_{t_{0}}^{\zeta} K\left[\zeta-z, g_{n-1}(z)\right] f_{n-1}(z) d z
\end{array}\right.
$$

with $f_{0}(\zeta)=g_{0}(\zeta)=0$. We wish to show that in a region $\left|\zeta-t_{0}\right|<a$ in $\Omega D$ this procedure converges uniformly and yields a pair of analytic functions

$$
f(\zeta)=\lim _{n \rightarrow \infty} f_{n}(\zeta), \quad g(\zeta)=\lim _{n \rightarrow \infty} g_{n}(\zeta)
$$

satisfying (6.7).

A few preliminaries are required before we proceed to the proof. We notice that near $t_{0}$ the level curves $u=\epsilon, \epsilon>0$, can be represented by giving $y$ as an at most two-valued function of $x$, in view of the symmetrized shape which $D$ must assume according to the beginning of Section 5 . In fact, symmetrization of $D$ and of the level curves of $u$ in the $x$-axis, as carried through by Pólya \& Szegö [17], would diminish $\lambda A$ if this were not the case. It follows that the pair of functions $v$ and $w$ of equation (2.10) with $x_{0}=\operatorname{Re}\left\{t_{0}\right\}$ yield a one-to-one map of the neighborhood of $t_{0}$ in $D$. As a result, $v+i w$ is continuous, as was shown in Section 2 .

We learn from (5.4) that for a suitable sequence of positive values $\epsilon_{n} \rightarrow 0$, the level curves $u=\epsilon_{n}$ have uniformly bounded lengths. Suppose that $t_{0}$ is a point where the tangent of $C$ is defined, so that (5.7) holds, and let $\Gamma$ be a segment in $D$ ending in $t_{0}$. Then along $\Gamma$ and along the level curves $u=\epsilon_{n}$, for given $\eta>0$,

$$
\int\left|\frac{\partial u}{\partial z}\right||d z| \leqq \eta
$$

in a sufficiently small neighborhood $\Delta$ of $t_{0}$. Such an estimate is immediate along $\Gamma$, and along the level curves $u=\epsilon_{n}$ it follows from the continuity near $t_{0}$ of the function $w$ of (2.10) and the identity

$$
\begin{aligned}
2\left|\frac{\partial u}{\partial z}\right| \cos \left[\lambda^{\frac{3}{2}}\left(x-x_{0}\right)\right] & =\frac{\partial u}{\partial \nu} \cos \left[\lambda^{\frac{1}{2}}\left(x-x_{0}\right)\right] \\
& =-\frac{\partial w}{\partial s}+u \frac{\partial}{\partial \nu} \cos \left[\lambda^{\frac{1}{2}}\left(x-x_{0}\right)\right],
\end{aligned}
$$

where $\nu$ and $s$ are the normal and arc length along the level curve.

The path $\Gamma$ and the discrete set of level curves $u=\epsilon_{n}$ do not cover a complete neighborhood of $t_{0}$ in $D$. In order to obtain paths which do have this property 
and for which (6.9) is also valid, but which, moreover, have bounded lengths, we note that by (5.8) $|\partial u / \partial z| \leqq M_{0} / R^{\frac{1}{2}}$, where $R$ is the shortest distance from $z$ to $C$ and where $M_{0}$ is a suitable large constant independent of $z$. Thus along a ray from $\zeta$ to the point on $C$ nearest to $\zeta,(6.9)$ is valid with $\eta=2 M_{0} R^{\xi}$. Such a ray from $\zeta$ must intersect the curves $u=\epsilon_{n}$ for large $n$ and hence for small enough $\epsilon_{n}$, these rays, the path $\Gamma$, and the level curves $u=\epsilon_{n}$ generate a system of curves connecting $t_{0}$ to every point $\zeta$ in a neighborhood $\Delta$ of $t_{0}$ and satisfying (6.9) with $\eta$ replaced by $2 \eta$.

Thus we can assert that, for small enough $a>0$, there are paths $c(\zeta)$ in the region $\Delta(a)$ consisting of the intersection of $D$ with the circle $\left|\zeta-t_{0}\right|<a$ which have lengths bounded by a fixed number $L$, which join $t_{0}$ to $\zeta$, and along which

$$
\int_{t_{0}}^{\zeta}\left|u_{z}\right||d z| \leqq \eta(a)
$$

with $\eta(a) \rightarrow 0$ as $a \rightarrow 0$. To each point $\zeta \varepsilon \Delta(a)$ we associate a fixed path $c(\zeta)$ of the character described and the number

$$
\rho_{n}(\zeta)=\int_{t_{0}}^{\zeta}\left|f_{n}(z)\right||d z|
$$

with integration carried out along $c(\zeta)$. We set

$$
\rho_{n}=\max _{\zeta \varepsilon \Delta(a)} \rho_{n}(\zeta)
$$

We can now return to the rigorous discussion of the system of integral equations (6.7), using the paths $c(\zeta)$ in all integrations.

Since $J_{0}^{\prime}(0)=0$, we can find a number $M>0$ such that

$$
|K(\zeta-z, t)|=\left|\frac{1}{2} \lambda^{\frac{1}{2}} J_{0}^{\prime}\left\{\lambda^{\frac{3}{3}}[(z-\zeta) t]^{\frac{1}{2}}\right\}\left(\frac{t}{z-\zeta}\right)^{\frac{1}{2}}\right| \leqq M s
$$

for $|z-\zeta| \leqq 2 a,|t| \leqq s$. Let us suppose finally that $\left|g_{n-1}(\zeta)\right|<s, \zeta \varepsilon \Delta(a)$. Then we have the estimate

$$
\left|\int_{t_{0}}^{\zeta} K\left[\zeta-z, g_{n-1}(z)\right] f_{n-1}(z) d z\right| \leqq M s \rho_{n-1} .
$$

From (6.8) we conclude by means of (6.10) and (6.13)

$$
\left|\int_{t_{0}}^{z} f_{n}(z)^{2} d z\right| \leqq\left|\int_{t_{0}}^{z} u_{z}^{2} d z\right|+2 M s \rho_{n-1} \eta(a)+M^{2} s^{2} \rho_{n-1}^{2} L .
$$


Since we know from (5.10) that $\int_{t_{0}}^{z} u_{z}^{2} d z$ is continuous, this inequality leads to an estimate for $g_{n}(z)$. However, we have to derive some information about $\rho_{n-1}$ before we will be able to assert that $\left|g_{n}(\zeta)\right|<s$, which is our next aim. From (6.8) and (6.13). we obtain

$$
\rho_{n} \leqq \eta(a)+M s \rho_{n-1} L .
$$

Let us assume that analogous inequalities had also been established for all indices less than $n$. Then $\rho_{n}$ is surely less than the elements of the recursion

$$
r_{n}=\eta(a)+M s r_{n-1} L, \quad r_{0}=0,
$$

which can be calculated explicitly. One finds

$$
r_{n}=\eta(a) \frac{1-(M L s)^{n}}{1-M L s}
$$

We have to choose $s$ so small that $M L s<1$. Then we can estimate all values $\rho_{n}$ by the one inequality

$$
\rho_{n}<\eta(a) \frac{1}{1-M L s}
$$

We can now obviously choose $a$ so small that from (6.8) and (6.14) must follow $\left|g_{n}(\zeta)\right|<s$ in $\Delta(a)$. After this choice of $a$, we are guaranteed that the estimate

$$
\left|g_{n}(\zeta)\right|<s
$$

will hold for all values of $n$, and the estimates (6.13) and (6.18) are also valid for each integer $n$. From (6.8) and (6.13), we also deduce

$$
\left|f_{n}(\zeta)\right| \leqq\left|u_{\zeta}\left(\zeta, \bar{t}_{0}\right)\right|+\frac{M s}{1-M L s} \cdot \eta(a) .
$$

Having given bounds for the approximating functions $g_{n}(\zeta)$ and $f_{n}(\zeta)$, we proceed now to prove their uniform convergence. We introduce, for this purpose, the numbers

$$
\phi_{n}=\max _{\zeta \varepsilon \Delta(a)}\left|f_{n}(\zeta)-f_{n-1}(\zeta)\right|, \quad \gamma_{n}=\max _{\zeta \varepsilon \Delta(a)}\left|g_{n}(\zeta)-g_{n-1}(\zeta)\right|
$$

From (6.8), (6.10), (6.18), (6.19), and (6.20) we derive the inequalities

$$
\begin{gathered}
\gamma_{n} \leqq \frac{8 A}{\lambda} \phi_{n} \frac{\eta(a)}{1-M L s}, \\
\phi_{n} \leqq B_{1} s \phi_{n-1}+B_{2} \eta(a) \gamma_{n-1},
\end{gathered}
$$


with appropriate positive constants $B_{1}$ and $B_{2}$. We can choose $s$ and $a$ so small that

$$
\phi_{n} \leqq \tau \phi_{n-1}, \quad \gamma_{n} \leqq \phi_{n}, \quad \tau<1,
$$

which shows that $\phi_{n}$ and $\gamma_{n}$ converge to zero like the terms of a geometric series with a factor $<1$. Hence we proved that $f_{n}(\zeta)$ and $g_{n}(\zeta)$ converge uniformly in a sufficiently small subdomain $\Delta(a)$ of $D$ to analytic functions $f(\zeta)$ and $g(\zeta)$, respectively. These limit functions satisfy in $\Delta(a)$ the integral equations (6.7).

We compare now the equations (6.7) with the two identities (5.11) and (6.5). We choose a path of integration $c(\zeta)$ in $\Delta(a)$ issuing from the boundary point $t_{0}$ and lying so near to the boundary $C$ of $D$ that on $c(\zeta)$ the estimates

$$
\begin{gathered}
|u|<\epsilon, \\
\left|\int_{t_{0}, \bar{i}_{0}}^{t, \bar{t}}\left[\left(\frac{\partial u}{\partial z}\right)^{2} d z-\frac{\lambda u^{2}}{4} d \bar{z}\right]+\frac{\lambda}{4 A}\left(\bar{t}-\bar{t}_{0}\right)\right|<\epsilon
\end{gathered}
$$

are valid, for arbitrary $\epsilon>0$. We can choose $a>0$ so small that $\Lambda_{c}=\max$ (diameter of $c, \int_{t_{0}}^{\zeta}\left|u_{z}\right||d z|, \int_{t_{0}}^{\zeta}|f||d z|$ ) is as small as we please, where the integrals are extended over the path $c=c(\zeta)$.

We want to derive estimates for the differences $F(\zeta)=f(\zeta)-u_{\zeta}(\zeta, \bar{\zeta}), G(\zeta)=$ $g(\zeta)-\bar{\zeta}+\bar{t}_{0}$ on the curve $c$. Subtracting (6.5) and (5.11) from the corresponding equations (6.7), we obtain

$$
F(\zeta)=\int_{t_{0}}^{\zeta}\left\{K(\zeta-z, g(z)) f(z)-K\left(\zeta-z, \bar{z}-\bar{t}_{0}\right) u_{z}(z, \bar{z})\right\} d z
$$

$$
+\int_{i_{0}}^{\bar{\zeta}} \frac{\partial^{2} J_{0}}{\partial \bar{z} \partial \zeta} u(z, \bar{z}) d \bar{z}
$$

$$
\begin{aligned}
G(\zeta)=-\frac{4 A}{\lambda} \int_{t_{0}}^{\zeta}\left\{f(z)^{2}\right. & \left.-u_{z}(z, \bar{z})^{2}\right\} d z-A \int_{i_{0}}^{\bar{\zeta}} u(z, \bar{z})^{2} d \bar{z} \\
& -\frac{4 A}{\lambda}\left[\int_{t_{0}, i_{0}}^{5, \bar{\zeta}}\left\{u_{z}^{2} d z-\frac{\lambda u^{2}}{4} d \bar{z}\right\}+\frac{\lambda}{4 A}\left(\bar{\zeta}-\bar{t}_{0}\right)\right]
\end{aligned}
$$

We set

$$
F_{c}=\max _{\zeta \varepsilon c}|F(\zeta)|, \quad G_{c}=\max _{\zeta \varepsilon c}|G(\zeta)|
$$


and we derive easily from (6.27) and (6.28), using (6.25), (6.26), and (6.12) the following estimates similar to (6.22) and (6.23):

$$
\begin{gathered}
F_{c} \leqq \Lambda_{c}\left(B_{3} F_{c}+B_{4} G_{c}\right)+B_{5} \epsilon \\
G_{c} \leqq \Lambda_{c}\left(B_{6} F_{c}\right)+B_{7} \epsilon,
\end{gathered}
$$

where $B_{3}, \cdots, B_{7}$ are appropriate positive constants. We can chpose $a>0$, and hence $\Lambda_{c}$, so small that (6.29) and (6.30) imply, for suitable choice of $B_{8}$ and $B_{9}$,

$$
F_{c}<B_{8} \epsilon, \quad G_{c}<B_{9} \epsilon .
$$

The number $\epsilon>0$ can be chosen as small as we please by taking the path $c=c(\zeta)$ in $\Delta(a)$ sufficiently near to the boundary $C$ of $D$. Hence (6.31) implies that for points $t$ on $C$ in the circle $\left|t-t_{0}\right|<a$, we have

$$
\lim _{\zeta \rightarrow t} g(\zeta)=\bar{t}-\bar{t}_{0}
$$

This condition is merely a rigorous formulation of (6.1), and it implies that the curve $C$ is analytic in the neighborhood of $t_{0}$, by virtue of the developments based on the similar condition (4.12) in Section 4. One checks easily that the neighborhoods $\Delta(a)$ cover $C$ completely for a suitable set of choices of $t_{0}$, and hence the extremal membrane $D$ for (5.1) is bounded by analytic arcs in the region between $C_{1}$ and $C_{2}$.

We can now reformulate condition (5.7) to obtain on $C$ between $C_{1}$ and $C_{2}$ the identity

$$
\left(\frac{\partial u}{\partial \nu}\right)^{2}=\frac{\lambda}{A}
$$

Since $C$ is composed of analytic arcs, we would be justified in deducing (6.33) directly from the classical variation formula (3.4) and the corresponding expression

$$
\delta A=-\int_{c} \delta \nu d s
$$

for area. Our principal result, then, is the conclusion that $C$ exists and is analytic, so that a formal discussion of (5.1) based on (3.4) and (6.34) is justified.

We may apply the variational formulas (3.4) and (6.34) to those parts of $C$ which consist of arcs of $C_{1}$ and $C_{2}$. On $C_{1}$, we may perform variations with $\delta \nu \leqq 0$ and still obtain domains $D$ which contain $D_{1}$ and are contained in $D_{2}$. Because of (5.1), we have therefore for the minimum domain $D$ the condition

$$
\left(\frac{\partial u}{\partial \nu}\right)^{2} \leqq \frac{\lambda}{A}
$$


on the part of $C$ which belongs to $C_{1}$ and, similarly, we find

$$
\left(\frac{\partial u}{\partial \nu}\right)^{2} \geqq \frac{\lambda}{A}
$$

on that part of $C$ which belongs to $C_{2}$.

If we had started with a domain $D_{1}$, with area $A_{1}$ and principal frequency $\lambda_{1}$, such that along $C_{1}$ we had always the inequality

$$
\left(\frac{\partial u}{\partial \nu}\right)^{2} \leqq \frac{\lambda_{1}}{A_{1}}
$$

for the first eigenfunction $u$ of $D_{1}$, then the domain $D_{1}$ might possibly have been the minimum domain of the problem (5.1). Analogously, we can obtain $D_{2}$ as the minimum domain only if its first eigenfunction satisfies on $\mathrm{C}_{2}$

$$
\left(\frac{\partial u}{\partial \nu}\right)^{2} \geqq \frac{\lambda_{2}}{A_{2}},
$$

where $A_{2}, \lambda_{2}$ are the area and principal frequency of $D_{2}$.

7. Further applications of the method. We have shown in this paper the existence of analytic arcs solving the two extremal problems (4.1) and (5.1). Many further specific problems could be treated by the general methods used to analyze (4.1) and (5.1). For example, given a fixed arc $\Gamma$, we could show the existence of an analytic arc $C$ joining the end-points of $\Gamma$ and bounding with $\Gamma$ a membrane $D$ for which

$$
\lambda_{1} L^{2}=\text { minimum }
$$

Or, for suitable constants $\alpha>0, \beta>0$ and for a given closed curve $C_{1}$, we could show the existence of a piece-wise analytic curve $C$, not a circle, enclosing $C_{1}$ and bounding a membrane $D$ for which

$$
\lambda_{1}{ }^{\alpha} A^{\beta}=\text { minimum. }
$$

In another field, we could solve the following cavitational flow problem. Given an arc $y=f(x)>0$ in the interval $a<x<b$ with $f(a)=f(b)=0$, we ask for an irrotational axially symmetric flow of incompressible fluid past the volume of revolution $B$ obtained by rotating this arc about the $x$-axis; we consider the case in which a steam cavity $W$ has developed near $B$. We set up the flow problem as an extremal problem [6, 8] of the type (5.1) by asking that $W$ be bounded by the curve $y=f(x)$ and another curve $y=h(x) \geqq f(x)$ in the interval $c \leqq x \leqq d$, $a<c<d<b$, with $h(c)=f(c), h(d)=f(d)$, where $f$ is fixed and $h$ is chosen so that

$$
M+E-\mu V=\text { minimum. }
$$


Here $M$ is the virtual mass of the object $B+W, E$ is the potential energy term,

$$
E=2 \pi \int_{c}^{d} \int_{0}^{h(x)} g x y d y d x
$$

where $g$ is related to the density and the gravitational constant, $V$ is the volume,

$$
V=2 \pi \int_{c}^{d} \int_{0}^{h(x)} y d y d x
$$

and $\mu$ is a fixed parameter, the cavitation constant. More precisely, we denote by $\phi$ and $\psi$ the velocity potential and the stream function of the flow, with the behavior

$$
\phi=x+\frac{x \text { const. }}{\left(x^{2}+y^{2}\right)^{\frac{3}{2}}}+\cdots, \psi=\frac{y^{2}}{2}-\frac{y^{2} \text { const. }}{\left(x^{2}+y^{2}\right)^{\frac{3}{2}}}+\cdots
$$

at infinity and satisfying the system of partial differential equations

$$
\phi_{x}=\psi_{y} / y, \quad \phi_{y}=-\psi_{x} / y
$$

throughout the flow region. The virtual mass $M$ is given by the integral

$$
M=\pi \iint\{\nabla \phi-\nabla x\}^{2} y d y d x
$$

extended over the flow region.

The technique developed in this paper allows us to deduce the existence of an analytic extremal curve $y=h(x)$ for (7.3). Hence we are justified in applying the formal variational identities

$$
\begin{aligned}
& \delta V=2 \pi \int y \delta \nu d s \\
& \delta E=2 \pi g \int x y \delta \nu d s \\
& \delta M=\pi \int\left\{\frac{1}{y^{2}}\left(\frac{\partial \psi}{\partial \nu}\right)^{2}-1\right\} y \delta \nu d s
\end{aligned}
$$

analogous to (3.4), to the problem (7.3). Therefore we deduce that along the $\operatorname{arcs}$ of $y=h(x)$ on which $h(x)>f(x)$

$$
\frac{1}{2 y^{2}}\left(\frac{\partial \psi}{\partial \nu}\right)^{2}+g x=\mu+\frac{1}{2}
$$


since

$$
\delta M+\delta E-\mu \delta V=0
$$

for shifts $\delta \nu$ of those arcs. But (7.5) is, by Bernoulli's equation, exactly the condition required in order that the region $W$ between the curves $y=f(x)$ and $y=h(x)$ can be interpreted physically to be a steam cavity generated by the high velocity of the flow. Equation (7.5) states that the pressure is constant on the surface separating the steam from the water. This existence theorem, based on (7.3), is more general than those given earlier $[6,8]$ in that the flow takes place in a gravity field acting in the direction of the axis of symmetry, and the fixed boundary is assumed merely to have the form $y=f(x)$. In the preceding papers it was assumed that the body $B$ had also a symmetry in the $y$-axis, which restricted considerably the range of the existence proof.

Certain parts of our theory can be extended to partial differential equations of higher order than the second. A complete discussion would carry us too far, but a few miscellaneous remarks in this direction may prove to be sufficient. Let

$$
\Gamma(z, \bar{z} ; t, \bar{t})=(z-t)(\bar{z}-\bar{t}) \log (z-t)(\bar{z}-\bar{t})+\cdots
$$

be the Green's function in $D$ of the biharmonic equation

$$
\Delta \Delta \Gamma=0
$$

satisfying the boundary conditions of the clamped plate

$$
\Gamma=\frac{\partial \Gamma}{\partial \nu}=0
$$

on $C$. We ask for the clamped plate $D$, enclosing the origin, for which the following dimensionless combination of area $A$ and deflection $\Gamma=\Gamma(0,0 ; 0,0)$ is a minimum:

$$
\frac{A}{\Gamma}=\text { minimum. }
$$

For an extremal domain, the variational condition on the boundary is

$$
(\Delta \Gamma)^{2}=16 \pi \Gamma / A=16 c^{2},
$$

since it is easily shown that

$$
\delta A=-\int_{C} \delta \nu d s, \quad \delta \Gamma(0,0 ; 0,0)=-\frac{1}{16 \pi} \int_{C}|\Delta \Gamma(z, \bar{z} ; 0,0)|^{2} \delta \nu d s .
$$


On the other hand, we have by (7.7), on $C, \Gamma_{z} \equiv 0$, so that $\Gamma_{z z} \dot{z}+\Gamma_{z \bar{z}} \overline{\dot{z}}=0$ there. Hence on $C$

$$
\Gamma_{z z}=-c \overline{\dot{z}}^{2}
$$

But we can write

$$
\Gamma=\bar{z} F(z)+G(z)+z \overline{F(z)}+\overline{G(z)},
$$

with $G(z)$ analytic in $D$ and $F(z)$ analytic there except for a singularity $F(z)=$ $z \log z+\cdots$ at the origin. Thus

$$
\Gamma_{z z}=\bar{z} F^{\prime \prime}(z)+G^{\prime \prime}(z)
$$

and our variational condition can be written

$$
c \overline{\dot{z}}^{2}+\bar{z} F^{\prime \prime}(z)+G^{\prime \prime}(z)=0 .
$$

If we denote by $g(z)$ the solution in $D$ of the linear ordinary differential equation

$$
c \frac{d g}{d z}+g F^{\prime \prime}(z)+G^{\prime \prime}(z)=0
$$

we find by the uniqueness theorem for the solution that $g(z)=\bar{z}$ on $C$. Thus $C$ is, as usual, an analytic curve. Furthermore, since $G^{\prime \prime}$ is regular and $F^{\prime \prime}$ is regular except for a simple pole of residue 1 at the origin in $D$, we see that $g$ is regular except for an isolated singularity at the origin, where it has the development $g(z)=z^{-1 / c}+\cdots$. Since $g(z)=\bar{z}$ on $C$, it must be single-valued in $D$ and, hence, $1 / c=n$ is an integer. By definition $c^{2}=\pi \Gamma / A$, and since $D$ is the extremal domain for (7.8), we have for any domain the inequality

$$
A / \Gamma \geqq \pi n^{2} \text {. }
$$

Let us consider the least favorable case, namely $n=1$. In this case, $g(z)$ has a simple pole with residue 1 at the origin. Thus

$$
\Phi(z)=g(z)+z, \quad \Psi(z)=g(z)-z
$$

are easily seen to be schlicht conformal mappings of $D$ onto the entire plane slit along a horizontal or vertical segment. Let $t=t(z)$ be the map of $D$ onto a circle about the origin with $t(0)=0$ and $t^{\prime}(0)=1$; then clearly

$$
\Phi=\frac{1}{t}+t, \quad \Psi=\frac{1}{t}-t
$$

Hence

$$
z=\frac{1}{2}[\Phi-\Psi]=t
$$

which shows that $D$ must be a circle.

In the case of the unit circle one checks immediately that $A / \Gamma=\pi$. Thus the above integer $n$ turns out, indeed, to be one. 

tion

We could consider also the eigenvalues $k_{1}, k_{2}, \cdots$, of the clamped plate equa-

$$
\Delta \Delta u=k^{4} u
$$

and attempt to minimize $k_{1}^{2} A$, a classical problem of Rayleigh [18, 21]. Here analyticity of the extremal boundary can be discussed by means of the representation $u=v+w$ of $u$ as the sum of solutions $v$ and $w$ of the second order equations $\Delta v=k^{2} v, \Delta w+k^{2} w=0$, to which our analysis of (2.1) can be applied.

\section{REFERENCES}

[1] Carathéodory, C., Conformal representation, Cambridge, 1932.

[2] Courant, R., "Beweis des Satzes, dass von allen homogenen Membranen gegebenen Umfanges und gegebener Spannung die kreisförmige den tiefsten Grundton besitzt," Math. Zeit., 1 (1918), 321-328.

[3] Courant, R. \& Hilbert, D., Methoden der mathematischen Physik, 2 vols. Berlin, $1931-7$.

[4] Faber, G., "Beweis, dass unter allen homogenen Membranen von gleicher Fläche . . . ," Sitzber. Bayer. Akad., 1923, pp. 169-172.

[5] Garabedian, P. R., "Distortion of length in conformal mapping," Duke Math. J., 16 (1949), 439-459.

[6] Garabedian, P. R., LewY, H., \& Schiffer, M., "Axially symmetric cavitational flow," Ann. Math., to appear.

[7] Garabedian, P. R. \& Schiffer, M., "Identities in the theory of conformal mapping," Trans. Amer. Math. Soc., 65 (1949), 187-238.

[8] Garabedian, P. R. \& Spencer, D. C., "Extremal methods in cavitational flow," $J$. of Rational Mech. and Analysis, 1 (1952), 359-409.

[9] Hadamard, J., "Mémoire sur le problème d'analyse relatif à l'équilibre des plaques élastiques encastrées," Mémoirs des Savants Étrangers, 33 (1908).

[10] Kornhauser, E. T. \& Stakgold, I., "Application of variational methods to the equation $\nabla^{2} u+\lambda u=0$," Tech. Report No. 117, Harvard Univ., Cambridge, 1950.

[11] KraHN, E., "Über eine von Rayleigh formulierte Minimaleigenschaft des Kreises," Math. Ann., 94 (1924), 97-100.

[12] Lewy, H., "On minimal surfaces with partially free boundary," Comm. on Pure and Applied Math., 4 (1951), 1-13.

[13] - "A note on harmonic functions and a hydrodynamical application," Proc. Amer. Math. Soc., 3 (1952), 111-113.

[14] tions," to appear.

[15] Pockels, F., Über die partielle Differentialgleichung $\Delta u+k^{2} u=0$ und deren Auftreten in der mathematischen Physik, Leipzig, 1891.

[16] P6LYA, G., "Remarks on the foregoing paper," to appear.

[17] Pólya, G. \& Szegö, G., Isoperimetric inequalities of mathematical physics, Princeton, 1951.

[18] Rayleigh, J. W., The Theory of Sound, 2 Vols., 2nd ed., Cambridge, 1894-6.

[19] Schiffer, M., "The span of multiply-connected domains," Duke Math. J., 10 (1943), 209-216.

[20] — "Hadamard's formula and variation of domain functions," Amer. J. Math., 68 (1946), 417-448.

[21] Szegö, G., "On membranes and plates," Proc. Nat. Acad. Sci., 36 (1950), 210-216.

[22] Weinstein, A., "Étude des spectres des équations aux dérivées partielles de la théorie des plaques élastiques," Mem. Sci. Math. no. 88 (1937). 